\title{
Computational and Spectral Discussion of Some Sulbstituited Chalcone Derivatives
}

\author{
Velayutham Shanmuga Vadivoo 1(D), Chithathoor Venugopal Mythili 1,*(D), Ramalingam \\ Balachander $^{2}$ (D), Natarajan Vijayalakshmi ${ }^{3}$ (D), Parimalaselvam Vijaya 1 (D) \\ 1 Department of Chemistry, Rani Anna Govt. College for Women (Affiliated to Manonmaniam Sundaranar University), \\ Palayapettai 627 008, Tirunelveli, Tamil Nadu, India \\ 2 Department of Chemistry, Periyar Arts College, Cuddalore 607 001, Tamil Nadu, India \\ 3 Department of Chemistry, Shree Raghavendra Arts and Science College, Keezhamoongiladi 608 602, Chidambaram, Tamil \\ Nadu, India \\ * Correspondence: vsvchemresearch@gmail.com (C.V.M.);
}

Scopus Author ID 8886486400

Received: 4.09.2021; Revised: 29.10.2021; Accepted: 2.11.2021; Published: 24.11.2021

\begin{abstract}
The substituted chalcone derivatives 1-7 have been synthesized, and spectroscopic characterization were done as done using the experimental FT-IR, UV-Vis, GC-MS, 1D NMR spectroscopy. The favored conformation of substituted chalcone 3 was predicted theoretically by geometry optimization structure selected geometrical parameters and molecular properties such as NBO, AIM, HOMO-LUMO, MEP surface, and atomic charges were derived from optimized structures. The ${ }^{1} \mathrm{H}$ and ${ }^{13} \mathrm{C}$ NMR spectral data had been additionally computed using the Gaussian-09 package and compared with the experimental values. The antibacterial and antifungal activity was derived by the disc diffusion method.
\end{abstract}

Keywords: computation; synthesis; spectral; chalcones; conformations; biological activity.

(C) 2021 by the authors. This article is an open-access article distributed under the terms and conditions of the Creative Commons Attribution (CC BY) license (https://creativecommons.org/licenses/by/4.0/).

\section{Introduction}

Chalcones are open-chain flavonoids with two aromatic rings attached by carbonyl groups and two $\alpha, \beta$-unsaturated carbon atoms [1-6]. They are well-known intermediates for synthesizing different types of heterocyclic compounds such as isoxazolines [7], pyrazolines [8-13], pyrazoles [14], pyridines [15], cyclohexenones [16], and oxadiazole [17] derivatives which could be potential drug candidates. It possesses acceptor and donor groups, which enhance their importance in various fields. Chalcone derivatives are promising compounds to be developed as their chromophore absorbs in the UV region from Sunscreen Agent. The nonlinear optical (NLO) properties of conjugated organic compounds have been the major focus of various computational and experimental studies because of their promising applications in photonics and optoelectronics, integrated optics, photophysical, high-speed optical communications, optical data processing, and storage $[18,19]$. The chalcone compounds have high significance because of their excellent NLO properties [20]. Chalcones are intramolecular charge transfer molecules, which allow one to design them based on the above design criteria. Chalcones are well known for their excellent blue-light transmittance and high second harmonic generation (SHG) conversion efficiency [21]. These chalcones crystallize in a noncentrosymmetric crystal structure and provide a necessary configuration for NLO activity with two planar benzene rings connected through a conjugated double bond [22-24]. Most of the 
work reported on these chalcones is about SHG and hirshfeld surface analysis (HAS) [25-27]. The molecule must have an $\pi$-electron backbone connected to acceptors/ donators groups $[28,29]$, commonly designated lateral substituents. The size of the molecule could also be an essential parameter to improve the NLO response, but if one considers this as a blind parameter than the shortest molecules will always be at a disadvantage against the biggest ones. Indeed, if the dimensions of the -electron spine will increase, the NLO response commonly increases a great-linear feature [30,31]. Despite that, molecules with a short-electron spine period could also reap desirable NLO responses, depending on the kind and function of the lateral substituents used [32-36]. Chalcone derivatives are promising compounds to be developed as their chromophore absorbs in the UV region from Sunscreen Agent, photoprotective and antioxidant properties [37-40]. Generally, chalcones are pharmacological activities such as anti-inflammatory, antibacterial, anticancer [41], antimalarial, antiproliferative [42], antifungal, antiviral, antioxidant, antineoplastic [43]. Free radical scavenging properties of phenol groups of chalcones increased the interest in the consumption of plants that included chalcones. Being the precursors of all the other flavonoid groups, chalcones are very important biosynthetic compounds. Much research work has been done on the synthesis and reactions of the chalcones.

The present investigation focuses on the synthesis and theoretical investigation of the molecular structure, charges, NBO, and AIM analysis of some known substituted chalcones. HOMO-LUMO energies, MEP surface dipole moment, polarizability, and first hyperpolarizability were also determined by DFT method and analyzed.

\section{Materials and Methods}

\subsection{Synthesis of substituted chalcones 1-7.}

Substituted acetophenone $(0.01 \mathrm{~mol})$ and 4-methoxybenzaldehyde $(0.01 \mathrm{~mol})$ were dissolved in ethanol. The ethanolic reaction mixture is poured into an RB flask [44]. The mixture is kept in an ice bath and stirred using a magnetic stirrer. Then $10 \mathrm{~mL}$ of $2 \mathrm{~N} \mathrm{NaOH}$ solution is added dropwise to the reaction mixture and stirred vigorously for $30 \mathrm{~min}$. After vigorous stirring, the reaction mixture is transferred to a beaker and kept in a refrigerator overnight. Then the reaction mixture is neutralized using $\mathrm{HCl}$ in the cold condition where precipitation occurred. On filtering off, the yellow color crude chalcone is obtained and dried. The crude is purified using thin-layer chromatography, and it can be performed on an aluminum plate pre-coated with silica gel-G using ethyl acetate and $n$-hexane $(7: 3)\left(R_{f}=0.7\right)$ as the solvent, and the spot was exposed to iodine vapor. The pure product was recrystallized from ethanol. The physical data are: 1. m.p. $110{ }^{\circ} \mathrm{C}$, yield 90\%, GC-MS: $239.4\left[\mathrm{M}^{+}\right]$; 2. m.p. 115 ${ }^{\circ} \mathrm{C}$, yield 89\%, GC-MS: $253.3\left[\mathrm{M}^{+}\right.$]; 3. m.p. $119{ }^{\circ} \mathrm{C}$, yield 85\%, GC-MS: $267.5\left[\mathrm{M}^{+}\right], 268.4$ [M+1]; 4. m.p. $132{ }^{\circ} \mathrm{C}$, yield 90\%, GC-MS: $257.6\left[\mathrm{M}^{+}\right], 258.2[\mathrm{M}+2]$; 5. m.p. $135^{\circ} \mathrm{C}$, yield 90\%, GC-MS: $274.2\left[\mathrm{M}^{+}\right], 275.5$ [M+2]; 6. m.p. $128^{\circ} \mathrm{C}$, yield 90\%, GC-MS: 317.4 [M+1], 318.4 [M+2]; 7. m.p. $144{ }^{\circ} \mathrm{C}$, yield 85\%, GC-MS: $284.3\left[\mathrm{M}^{+}\right], 285.3[\mathrm{M}+2]$.

\subsection{Spectral measurements.}

The ${ }^{1} \mathrm{H}$ NMR (400 MHz) and ${ }^{13} \mathrm{C}$ NMR (100 MHz) had been recorded at room temperature on Bruker $400 \mathrm{MHz}$ instrument compounds dissolved for chloroform- $d_{6}$. The mass spectra were performed using Varian Saturn 2200 GC-MS spectrometers. 
FT-IR spectrometer and the wavenumbers are given in $\mathrm{cm}^{-1}$. The UV-visible spectra of the chalcones 1-7 are recorded in Shimadzu-UV-1800-UV-Visible spectrophotometer using N,Ndimethylformamide as solvent at ambient room temperature.

\subsection{Computational study.}

Geometry optimizations were carried out according to density functional theory using B3LYP/6-31G(d,p) basis set [45] in the Gaussian-09 package. The polarizabilities and hyperpolarizabilities have been derived from the DFT optimized structure by using finite discipline technique using the B3LYP/6-31G* basis set, while the NBO calculations were carried out using the premise set B3LYP/6-311+G(d,p) available in Gaussian-09 and AIM calculations were done using B3LYP/6-31G(d,p) basis set.

\section{Results and Discussion}

(E)-1-(4-methoxyphenyl)-3-phenylprop-2-en-1-one 1, (E)-1-(4-methoxyphenyl)-3-ptolylprop-2-en-1-one $\quad 2, \quad(E)-1,3-b i s(4-m e t h o x y p h e n y l) p r o p-2-e n-1-o n e \quad 3, \quad(E)-3-(4-$ fluorophenyl)- 1-(4-methoxyphenyl)prop-2-en-1-one 4, (E)-3-(4-chlorophenyl)-1-(4methoxyphenyl)prop-2-en-1-one 5, (E)-3-(4-bromophenyl)-1-(4-methoxyphenyl)prop-2-en-1one 6 and (E)-1-(4-methoxyphenyl)-3-(4-nitrophenyl)prop-2-en-1-one 7 were synthesized according to the Scheme 1 and characterized by GC-MS, IR, UV-Vis, ${ }^{1} \mathrm{H}$ and ${ }^{13} \mathrm{C}$ NMR spectra. The labeling of the atoms followed in the present study was also indicated in the Scheme 1.<smiles>[X]c1ccc(C(=O)[O-])cc1</smiles>
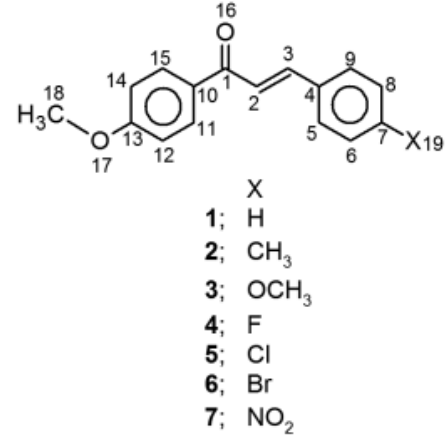

Scheme 1. Steps involved in the synthesis of chalcones 1-7.

\subsection{IR spectra.}

The intense peaks around $1664 \mathrm{~cm}^{-1}$ in the IR spectra of $1-7$ are presented to $v_{\mathrm{C}=\mathrm{O}}$ of the carbonyl group. The aromatic $v_{\mathrm{C}=\mathrm{C}}$ stretching vibrations are observed around $3129 \mathrm{~cm}^{-1}$, and the stretching vibrations of the aromatic $-\mathrm{CH}=$ out-of-plane bending vibrations have appeared around $854 \mathrm{~cm}^{-1}$. The $\alpha, \beta$-unsaturated $-\mathrm{C}=\mathrm{C}-$ chain are found to be $1212 \mathrm{~cm}^{-1}$. Compound 7 peaks for $v_{\mathrm{NO} 2}$ group are observed in regions 1505 and $1480 \mathrm{~cm}^{-1}$. The sharp peaks region $1086 \mathrm{~cm}^{-1}$ are attributed to $v_{\mathrm{C}-\mathrm{O}}$ mode. The appearance of peaks around $1268 \mathrm{~cm}^{-}$ ${ }^{1}$ is due to the presence of asymmetric $\mathrm{C}-\mathrm{O}-\mathrm{C}$ stretching vibration. The intense high peaks around 612 to $543 \mathrm{~cm}^{-1}$ are assigned to $\mathrm{C}-\mathrm{X}(\mathrm{X}=\mathrm{Fl}, \mathrm{Cl}$, and $\mathrm{Br})$ stretching vibration $[46,47]$. The remaining IR spectral data of 1-7 are listed in Table 1.

Table 1. IR spectral data $\left(\mathrm{cm}^{-1}\right)$ for $1-7$.

\begin{tabular}{l|l|l|l|l|l|l|l} 
Assignments & $\mathbf{1}$ & $\mathbf{2}$ & $\mathbf{3}$ & $\mathbf{4}$ & $\mathbf{5}$ & $\mathbf{6}$ & $\mathbf{7}$ \\
\hline$v_{\mathrm{C}=\mathrm{O}}$ & 1660 & 1654 & 1658 & 1655 & 1659 & 1664 & 1657 \\
\hline$v_{\mathrm{C}=\mathrm{C}}$ & 30452954 & 30282989 & 3073 & 3127 & 3025 & 3129 & 2980
\end{tabular}




\begin{tabular}{|c|c|c|c|c|c|c|c|}
\hline Assignments & 1 & 2 & 3 & 4 & 5 & 6 & 7 \\
\hline$v_{\mathrm{C}-\mathrm{O}}$ & 1023 & 1045 & 1016 & $\begin{array}{l}1018 \\
1034 \\
\end{array}$ & $\begin{array}{l}1086 \\
1031 \\
\end{array}$ & $\begin{array}{l}1083 \\
1019 \\
\end{array}$ & 1028 \\
\hline $\begin{array}{l}\text { Aromatic CHout-of-plane } \\
\text { bending vibration }\end{array}$ & $\begin{array}{ll}854 & 783 \\
743 & \end{array}$ & $\begin{array}{ll}808 & 787 \\
713 & \end{array}$ & $\begin{array}{l}817 \\
750 \\
- \\
\end{array}$ & $\begin{array}{l}825 \\
743 \\
- \\
\end{array}$ & $\begin{array}{l}878 \\
757 \\
723 \\
\end{array}$ & $\begin{array}{l}830 \\
777 \\
- \\
\end{array}$ & $\begin{array}{l}826 \\
773 \\
749 \\
\end{array}$ \\
\hline$v_{\mathrm{C}-\mathrm{O}-\mathrm{C}}$ & 1244 & 1264 & 1255 & 1259 & 1262 & 1262 & 1268 \\
\hline$v_{\mathrm{NO} 2}$ & - & - & - & - & - & - & $\begin{array}{l}1480 \\
1505 \\
1422 \\
\end{array}$ \\
\hline$\nu_{\mathrm{Cl}} / \nu_{\mathrm{Br}} / \nu_{\mathrm{F}}$ & - & - & - & $\begin{array}{l}534 \\
611\end{array}$ & 538 & $\begin{array}{l}612 \\
563\end{array}$ & - \\
\hline
\end{tabular}

\subsection{Electronic spectral studies.}

The ultraviolet absorption spectra were recorded for all the synthesized chalcones 1-7 in dimethylformamide region $260-400 \mathrm{~nm}$. The maximum absorption values around 347, 348, $358,360,363,350$, and $389 \mathrm{~nm}$ due to $\mathrm{n}-\pi^{*}$ transition and 280, 286, 275, 260, 290, 273 and $297 \mathrm{~nm}$ due to $\pi-\pi^{*}$ transition, which may be attributed the excitation in the $\mathrm{C}=\mathrm{O}$ and $-\mathrm{C}=\mathrm{C}-$ group [45]. The higher $\lambda_{\max }$ is observed for 7 when compared to the other chalcones $1-6$ due to the presence of $\mathrm{NO}_{2}$ moiety. UV-visible spectra of 3, 4, and 7 are shown in Figs. 1-3.

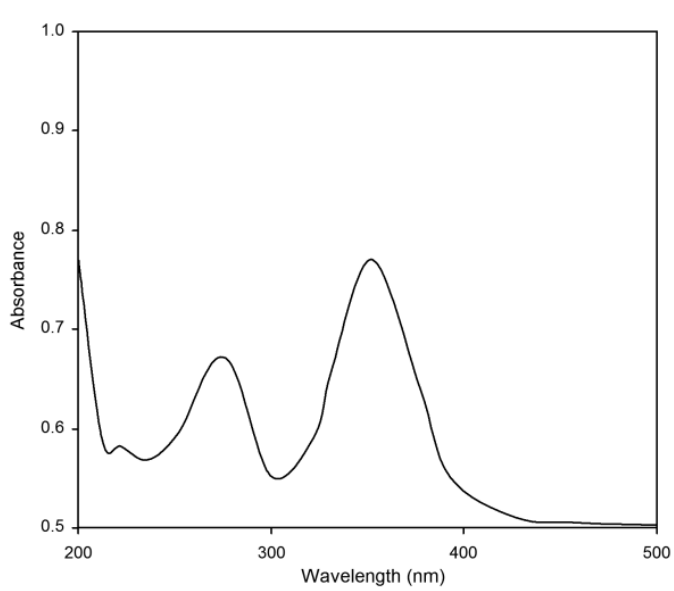

Figure 1. UV-Vis spectrum of 3 .

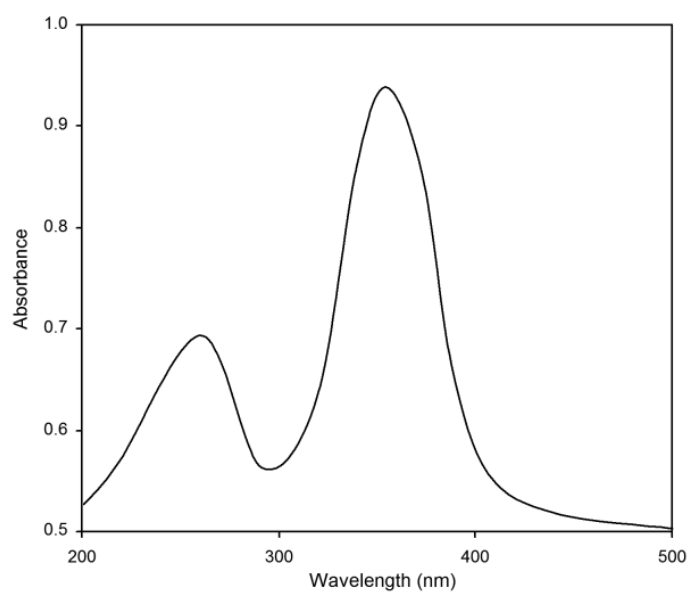

Figure 2. UV-Vis spectrum of 4 .

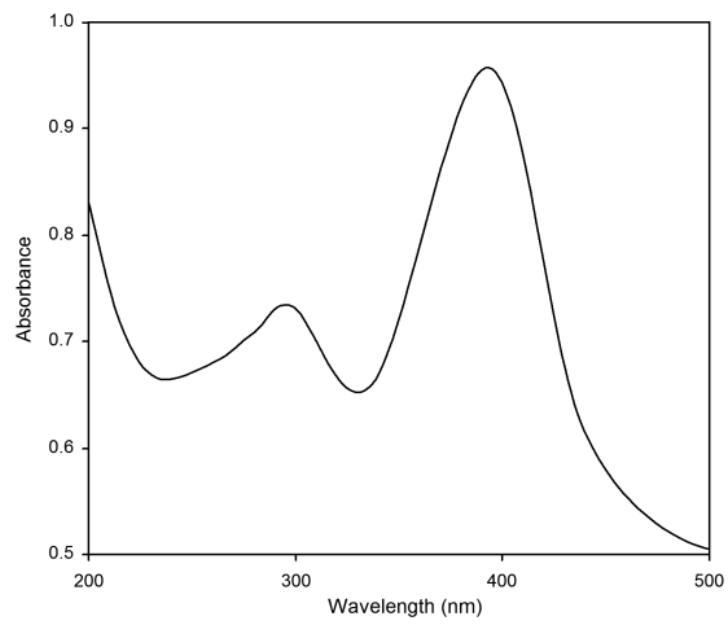

Figure 3. UV-Vis spectrum of 7.

\section{3. ${ }^{1} \mathrm{H}$ NMR spectral analysis.}

(E)-3-(4-Fluorophenyl)-1-(4-methoxyphenyl)prop-2-en-1-one 4, the signals in the ${ }^{1} \mathrm{H}$ NMR spectrum was assigned based on these positions, integrals, and multiplicities. The 
high-frequency doublet at $8.04 \mathrm{ppm}$ is assigned to $\mathrm{H}-11$ and $\mathrm{H}-15$ protons (meta position with respect methoxy and ortho position concerning carbonyl group with coupling constant $8.8 \mathrm{~Hz}$ ). The doublet of the doublet peak observed at $7.63 \mathrm{ppm}$ is assigned to $\mathrm{H}-5$ and $\mathrm{H}-9$ protons (integrated corresponding to two protons coupling constant $8.4 \mathrm{~Hz}$ ). The special category of fluorine substituted in aromatic rings is observed in ${ }^{1} \mathrm{H}$ NMR spectra as a doublet of the doublet. The remaining aromatic ring protons that appeared at 7.11 and $6.99 \mathrm{ppm}$ are represented $\mathrm{H}-$ 6/H-8 and $\mathrm{H}-12 / \mathrm{H}-14$. The two doublets seem at 7.76, and $7.47 \mathrm{ppm}$ (each doublet for one proton) are assigned to $\alpha$ and $\beta$ hydrogen of chalcone moiety at $\mathrm{H}-3$ and $\mathrm{H}-2$ protons with a coupling constant of $15.6 \mathrm{~Hz}$, which is characterized to geminal coupling constant of trans configuration. The ${ }^{13} \mathrm{C}$ NMR spectrum less intense peak observed at $187.5 \mathrm{ppm}$ is assigned to carbonyl carbon $\mathrm{C}-1$. There are four ipso carbons present in this molecule, and their peaks are observed in the spectra. A low-frequency signal at $123.71 \mathrm{ppm}$ is assigned to the quaternary carbon $\mathrm{C}-4$ since it is para concerning the electron-withdrawing fluorine group. The signal at $141.75 \mathrm{ppm}$ is assigned to the ipso carbon $\mathrm{C}-7$, attached to the fluorine atom $\mathrm{F}-19$. The two signals at 130.27 and $163.31 \mathrm{ppm}$ are due to $\mathrm{C}-10$, which is linked to carbonyl carbon $\mathrm{C}-1$ and C-13, respectively. The remaining aromatic ring carbons appeared at 114.04, 130.96, 130.63, and $131.83 \mathrm{ppm}$, respectively. In a similar manner, assignments were done for other chalcone derivatives $1-3$ and 5- 7. The ${ }^{1} \mathrm{H}$ and ${ }^{13} \mathrm{C}$ chemical shift values are obtained in Tables 2 and 3, the ${ }^{1} \mathrm{H}$ and ${ }^{13} \mathrm{C}$ NMR spectra of 4 as shown in Figs. 4 and 5, respectively.

Table 2. ${ }^{1} \mathrm{H}$ NMR chemical shifts (ppm) of 1-7.

\begin{tabular}{|c|c|c|c|c|c|c|c|c|}
\hline Compds. & H-2 & H-3 & H-5/H-9 & H-6/H-8 & H-11/H-15 & H-12/H-14 & H-18 & H-19 \\
\hline 1 & 7.46 & $\begin{array}{c}7.63 \\
(\mathrm{~d}, 15.0 \mathrm{~Hz})\end{array}$ & 7.51 & $\begin{array}{c}6.74 \\
(\mathrm{~d}, 8.8 \mathrm{~Hz})\end{array}$ & $\begin{array}{c}8.00 \\
(\mathrm{~d}, 8.8 \mathrm{~Hz})\end{array}$ & $\begin{array}{c}7.04 \\
(\mathrm{~d}, 8.0 \mathrm{~Hz})\end{array}$ & $3.56(\mathrm{~s})$ & - \\
\hline 2 & 7.49 & $\begin{array}{c}7.79 \\
(\mathrm{~d}, 15.6 \mathrm{~Hz})\end{array}$ & 7.53 & $\begin{array}{c}6.98 \\
(\mathrm{~d}, 8.8 \mathrm{~Hz}) \\
\end{array}$ & $\begin{array}{c}8.04 \\
(\mathrm{~d}, 8.8 \mathrm{~Hz})\end{array}$ & $\begin{array}{c}7.22 \\
(\mathrm{~d}, 8.0 \mathrm{~Hz})\end{array}$ & $3.89(\mathrm{~s})$ & $2.40(\mathrm{~s})$ \\
\hline 3 & 7.77 & 7.83 & 7.81 & $\begin{array}{c}7.02 \\
(\mathrm{~d}, 8.8 \mathrm{~Hz})\end{array}$ & $\begin{array}{c}8.14 \\
(\mathrm{~d}, 8.8 \mathrm{~Hz})\end{array}$ & $\begin{array}{c}7.08 \\
(\mathrm{~d}, 8.4 \mathrm{~Hz})\end{array}$ & $3.87(\mathrm{~s})$ & $3.82(\mathrm{~s})$ \\
\hline 4 & $\begin{array}{c}7.76 \\
(\mathrm{~d}, 15.6 \mathrm{~Hz}) \\
\end{array}$ & $\begin{array}{c}7.47 \\
(\mathrm{~d}, 15.6 \mathrm{~Hz})\end{array}$ & $\begin{array}{c}7.63 \\
(\mathrm{~d}, \mathrm{~d}, 8.4 \mathrm{~Hz})\end{array}$ & $\begin{array}{c}6.99 \\
(\mathrm{~d}, 8.8 \mathrm{~Hz})\end{array}$ & $\begin{array}{c}8.04 \\
(\mathrm{~d}, 8.8 \mathrm{~Hz})\end{array}$ & $7.11(\mathrm{t})$ & $3.90(\mathrm{~s})$ & - \\
\hline 5 & $7.83(\mathrm{~d})$ & $\begin{array}{c}7.95 \\
(\mathrm{~d}, 15.6 \mathrm{~Hz}) \\
\end{array}$ & $7.66(\mathrm{~m})$ & $7.66(\mathrm{~m})$ & $\begin{array}{c}8.16 \\
(\mathrm{~d}, 9.2 \mathrm{~Hz}) \\
\end{array}$ & $\begin{array}{c}7.09 \\
(\mathrm{~d}, 8.8 \mathrm{~Hz}) \\
\end{array}$ & $3.87(\mathrm{~s})$ & - \\
\hline 6 & $\begin{array}{c}8.74 \\
(\mathrm{~d}, 15.6 \mathrm{~Hz}) \\
\end{array}$ & $\begin{array}{c}7.43 \\
(\mathrm{~d}, 15.6 \mathrm{~Hz})\end{array}$ & $\begin{array}{c}7.60 \\
(\mathrm{~d}, 8.4 \mathrm{~Hz}) \\
\end{array}$ & $\begin{array}{c}7.67 \\
(\mathrm{~d}, 8.8 \mathrm{~Hz}) \\
\end{array}$ & $\begin{array}{c}8.17 \\
(\mathrm{~d}, 8.8 \mathrm{~Hz})\end{array}$ & $\begin{array}{c}7.04 \\
(\mathrm{~d}, 8.8 \mathrm{~Hz})\end{array}$ & $3.67(\mathrm{~s})$ & - \\
\hline 7 & $\begin{array}{c}7.67 \\
(\mathrm{~d}, 15.6 \mathrm{~Hz})\end{array}$ & $\begin{array}{c}7.88 \\
(\mathrm{~d}, 15.6 \mathrm{~Hz})\end{array}$ & $\begin{array}{c}7.09 \\
(\mathrm{~d}, 8.4 \mathrm{~Hz})\end{array}$ & $\begin{array}{c}8.17 \\
(\mathrm{~d}, 8.0 \mathrm{~Hz})\end{array}$ & 7.21 & $\begin{array}{c}7.09 \\
(\mathrm{~d}, 8.4 \mathrm{~Hz})\end{array}$ & $3.87(\mathrm{~s})$ & - \\
\hline
\end{tabular}

Values within parentheses are the observed coupling constants.

Table 3. ${ }^{13} \mathrm{C}$ NMR chemical shifts (ppm) of 1-7.

\begin{tabular}{|c|c|c|c|c|c|c|c|c|c|c|c|c|}
\hline Compds. & C-1 & $\mathrm{C}-2$ & $\mathrm{C}-\mathbf{3}$ & $C-4$ & $\begin{array}{l}\mathrm{C}-5 / \\
\mathrm{C}-9\end{array}$ & $\begin{array}{l}\mathrm{C}-6 / \\
\mathrm{C}-8\end{array}$ & C-10 & $\begin{array}{l}\mathrm{C}-11 / \\
\mathrm{C}-15\end{array}$ & $\begin{array}{l}\mathrm{C}-12 / \\
\mathrm{C}-14\end{array}$ & $\mathrm{C}-7$ & $\mathrm{C}-13$ & $\begin{array}{l}C-18 / \\
C-14\end{array}$ \\
\hline 1 & 185.73 & 115.47 & 138.71 & 126.73 & 110.67 & 130.27 & 123.73 & 112.96 & 130.65 & 130.33 & 158.71 & 55.41 \\
\hline 2 & 186.83 & 118.17 & 140.73 & 129.33 & 112.97 & 130.45 & 126.33 & 114.01 & 130.85 & 130.61 & 161.73 & 55.56 \\
\hline 3 & 187.36 & 119.46 & 143.14 & 127.38 & 113.95 & 130.60 & 127.38 & 114.37 & 130.74 & 161.20 & 163.06 & $\begin{array}{l}55.50 / \\
55.33\end{array}$ \\
\hline 4 & 187.35 & 122.81 & 134.05 & 123.71 & 130.96 & 114.04 & 130.27 & 131.83 & 130.63 & 141.75 & 163.31 & 55.56 \\
\hline 5 & 184.23 & 121.51 & 141.75 & 134.05 & 130.63 & 130.27 & 123.71 & 130.96 & 114.04 & 134.05 & 163.30 & 55.47 \\
\hline 6 & 187.33 & 121.73 & 142.83 & 134.11 & 130.30 & 130.17 & 122.89 & 130.45 & 113.86 & 134.00 & 163.05 & 55.73 \\
\hline 7 & 187.44 & 121.21 & 143.62 & 130.33 & 130.49 & 106.37 & 139.60 & 130.90 & 113.96 & 153.08 & 163.17 & 56.09 \\
\hline
\end{tabular}

\subsection{Conformational analysis.}

There are three possible conformations for the chalcone 3, as shown in Fig.6. In conformation A, both methoxy methyl moiety is syn to $\alpha, \beta$-unsaturated carbonyl group $\mathrm{C}=\mathrm{O}$ chain, whereas, in $\mathrm{B}$, it is anti. The conformation $\mathrm{C}$, one methoxy methyl is syn another methoxy methyl is anti to the carbonyl carbon. Theoretical study [relative energies are 0.22 
(A), $0.39(\mathrm{~B})$, and $0.00(\mathrm{C}) \mathrm{kcal} \mathrm{mol}^{-1}$ ] predicts the favored conformation as $\mathrm{C}$ only for the chalcone 3 .

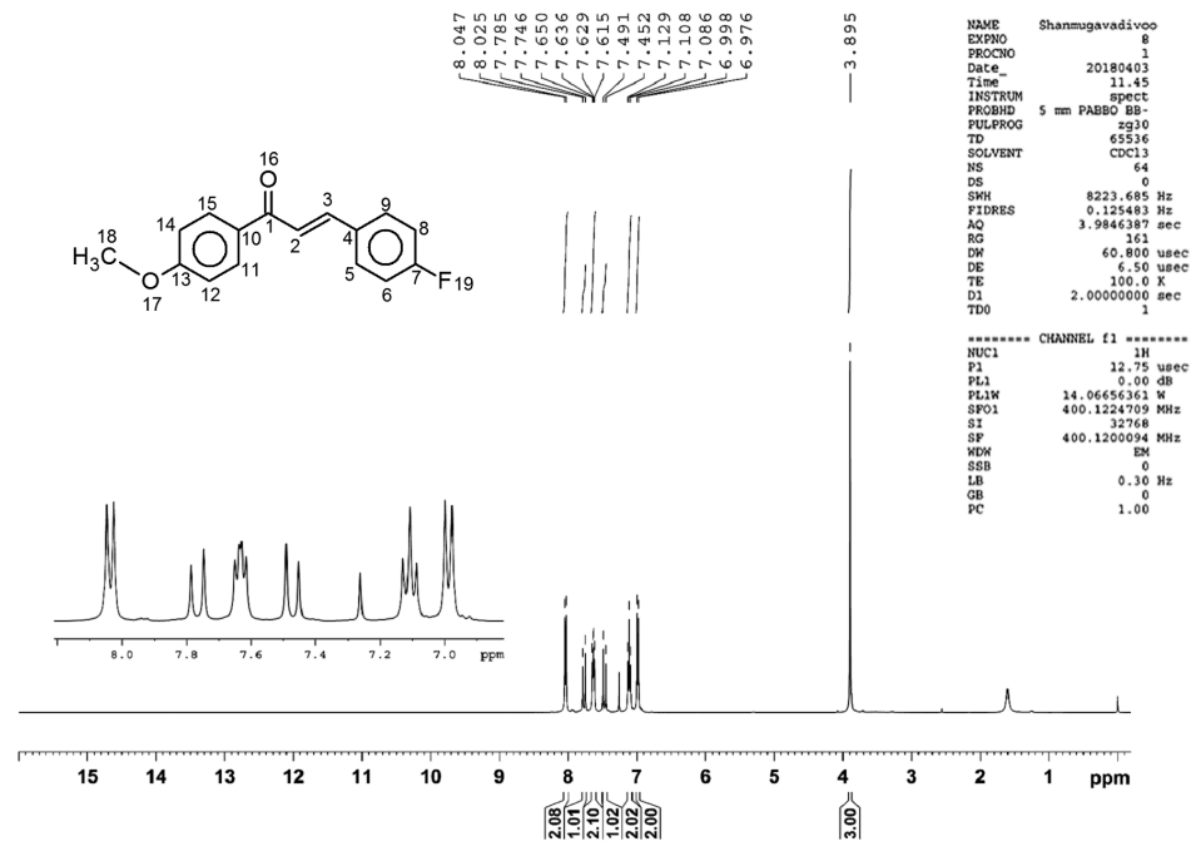

Figure $4{ }^{1} \mathrm{H}$ NMR spectrum of 4 .
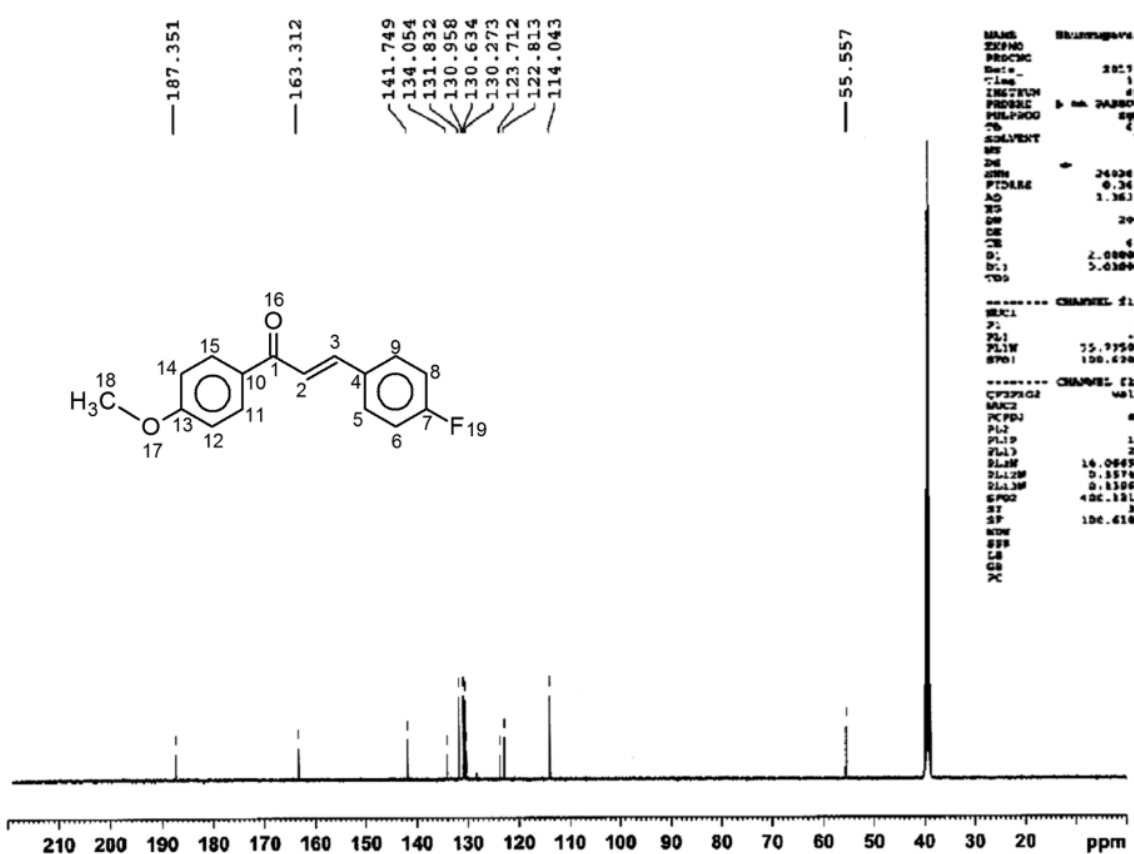

$\begin{array}{llllllllllllllllllllllll}210 & 200 & 190 & 180 & 170 & 160 & 150 & 140 & 130 & 120 & 110 & 100 & 90 & 80 & 70 & 60 & 50 & 40 & 30 & 20 & \text { ppm }\end{array}$

Figure 5. ${ }^{13} \mathrm{C}$ NMR spectrum of 4 .

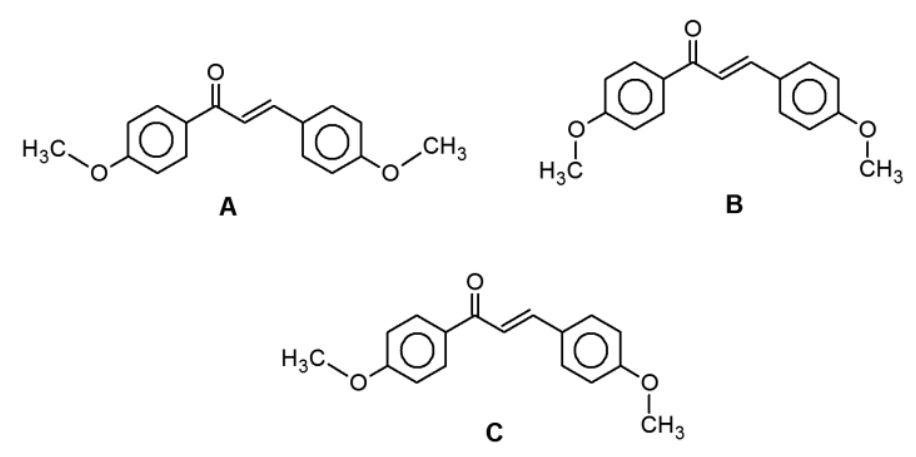

Figure 6. Possible conformations of 3. 


\subsection{Molecular properties.}

3.5.1. Geometric parameters.

The selected bond length, bond angle, and torsional angle values are derived from the corresponding optimized structures 1-7 are given in Table 4 . The torsional angle $\mathrm{C} 11-\mathrm{C} 10$ $\mathrm{C} 1-\mathrm{C} 2$ in chalcone $3\left(-0.35^{\circ}\right)$ differs from other chalcones $1-7\left(\approx 0.30^{\circ}\right)$ indicating that the $\alpha, \beta$-unsaturated $-\mathrm{C}=\mathrm{C}-$ chain slightly distorted from the other chalcones. Further, the nitro substituted chalcone torsional angle is a slight deviation from the other chalcones $1-6$. The optimized structures of chalcones 1-7 are shown in Fig. 7.

Table 4. Selected geometric parameters [bond lengths $(\AA)$, bond angles $\left(^{\circ}\right)$ and torsional angles $\left(^{\circ}\right)$ ] in $1-7$.

\begin{tabular}{l|l|l|l|l|l|l|l|} 
Geometric parameters & $\mathbf{1}$ & $\mathbf{2}$ & $\mathbf{3}$ & $\mathbf{4}$ & $\mathbf{5}$ & $\mathbf{6}$ & $\mathbf{7}$
\end{tabular}

\begin{tabular}{|c|c|c|c|c|c|c|c|}
\hline \multicolumn{8}{|c|}{ Bond lengths } \\
\hline $\mathrm{C} 1-\mathrm{O} 16$ & 1.22 & 1.22 & 1.22 & 1.22 & 1.22 & 1.22 & 1.22 \\
\hline $\mathrm{C} 1-\mathrm{C} 2$ & 1.48 & 1.48 & 1.48 & 1.48 & 1.48 & 1.48 & 1.48 \\
\hline $\mathrm{C} 2-\mathrm{C} 3$ & 1.34 & 1.34 & 1.34 & 1.34 & 1.34 & 1.34 & 1.34 \\
\hline C4-C5 & 1.39 & 1.39 & 1.39 & 1.39 & 1.39 & 1.39 & 1.39 \\
\hline C5-C6 & 1.40 & 1.40 & 1.40 & 1.40 & 1.40 & 1.40 & 1.40 \\
\hline C7-C8 & 1.48 & 1.48 & 1.48 & 1.48 & 1.48 & 1.48 & 1.48 \\
\hline C8-C9 & 1.39 & 1.39 & 1.39 & 1.39 & 1.39 & 1.39 & 1.39 \\
\hline C1-C10 & 1.50 & 1.50 & 1.50 & 1.50 & 1.50 & 1.50 & 1.50 \\
\hline $\mathrm{C} 10-\mathrm{C} 11$ & 1.40 & 1.40 & 1.40 & 1.40 & 1.40 & 1.40 & 1.40 \\
\hline $\mathrm{C} 11-\mathrm{C} 12$ & 1.50 & 1.50 & 1.50 & 1.50 & 1.50 & 1.50 & 1.50 \\
\hline $\mathrm{C} 12-\mathrm{C} 13$ & 1.41 & 1.41 & 1.41 & 1.41 & 1.41 & 1.41 & 1.41 \\
\hline C13-O17 & 1.39 & 1.39 & 1.39 & 1.39 & 1.39 & 1.39 & 1.39 \\
\hline O17-C18 & 1.40 & 1.40 & 1.40 & 1.40 & 1.40 & 1.40 & 1.40 \\
\hline C13-C14 & 1.35 & 1.35 & 1.35 & 1.35 & 1.35 & 1.35 & 1.35 \\
\hline C14-C15 & 1.42 & 1.42 & 1.42 & 1.42 & 1.42 & 1.42 & 1.42 \\
\hline $\mathrm{C} 15-\mathrm{C} 10$ & 1.40 & 1.40 & 1.40 & 1.40 & 1.40 & 1.40 & 1.40 \\
\hline $\mathrm{N} 19-\mathrm{O} 20 / 21$ & - & - & - & - & - & - & $\begin{array}{l}1.23 / \\
1.23\end{array}$ \\
\hline \multicolumn{8}{|c|}{ Bond angles } \\
\hline $\mathrm{O} 16-\mathrm{C} 1-\mathrm{C} 2$ & 120.92 & 120.92 & 120.92 & 120.92 & 120.92 & 120.92 & 120.92 \\
\hline $\mathrm{O} 16-\mathrm{C} 1-\mathrm{C} 10$ & 119.14 & 119.14 & 119.14 & 119.14 & 119.14 & 119.14 & 119.14 \\
\hline $\mathrm{C} 1-\mathrm{C} 2-\mathrm{C} 3$ & 120.37 & 120.37 & 120.37 & 120.37 & 120.37 & 120.37 & 120.37 \\
\hline $\mathrm{C} 2-\mathrm{C} 3-\mathrm{H} 3$ & 116.09 & 116.09 & 116.09 & 116.09 & 116.09 & 116.09 & 116.09 \\
\hline C4-C5-C6 & 121.36 & 121.36 & 121.36 & 121.36 & 121.36 & 121.36 & 121.36 \\
\hline C4-C9-C8 & 117.49 & 117.49 & 117.49 & 117.49 & 117.49 & 117.49 & 117.49 \\
\hline $\mathrm{C} 5-\mathrm{C} 6-\mathrm{C} 7$ & 117.82 & 117.82 & 117.82 & 117.82 & 117.82 & 117.82 & 117.82 \\
\hline C6-C7-X19 & 121.48 & 121.48 & 124.84 & 119.04 & 119.60 & 119.64 & 119.14 \\
\hline C7-C19/O19/H'19/H"/H"' & - & $\begin{array}{l}111.30 / \\
110.99 / \\
111.43\end{array}$ & 118.39 & - & - & - & $\begin{array}{c}117.68 \\
/ 117.63\end{array}$ \\
\hline $\mathrm{C} 7-\mathrm{C} 8-\mathrm{C} 9$ & 121.33 & 121.33 & 121.33 & 121.33 & 121.33 & 121.33 & 121.33 \\
\hline $\mathrm{C} 1-\mathrm{C} 10-\mathrm{C} 11$ & 124.46 & 124.46 & 124.46 & 124.46 & 124.46 & 124.46 & 124.46 \\
\hline $\mathrm{C} 11-\mathrm{C} 12-\mathrm{C} 13$ & 120.15 & 120.15 & 120.15 & 120.15 & 120.15 & 120.15 & 120.15 \\
\hline C12-C15-O17 & 115.89 & 115.89 & 115.89 & 115.89 & 115.89 & 115.89 & 115.89 \\
\hline C13-O17-C18 & 118.67 & 118.67 & 118.67 & 118.67 & 118.67 & 118.67 & 118.67 \\
\hline C13-C14-C15 & 119.52 & 119.52 & 119.52 & 119.52 & 119.52 & 119.52 & 119.52 \\
\hline C14-C15-C10 & 121.78 & 121.78 & 121.78 & 121.78 & 121.78 & 121.78 & 121.78 \\
\hline C15-C10-C1 & 117.78 & 117.78 & 117.78 & 117.78 & 117.78 & 117.78 & 117.78 \\
\hline $\mathrm{C} 10-\mathrm{C} 1-\mathrm{O} 16$ & 119.95 & 119.95 & 119.95 & 119.95 & 119.95 & 119.95 & 119.95 \\
\hline $\mathrm{N} 19-\mathrm{O} 20-\mathrm{O} 21$ & & - & - & - & - & - & 124.69 \\
\hline \multicolumn{8}{|c|}{ Torsional angles } \\
\hline $\mathrm{O} 16-\mathrm{C} 1-\mathrm{C} 2-\mathrm{H} 2$ & 179.9 & 179.9 & 179.9 & -180.0 & -180.0 & -180.0 & 179.9 \\
\hline $\mathrm{H} 3-\mathrm{C} 3-\mathrm{C} 2-\mathrm{H} 2$ & -180.0 & -180.0 & -180.0 & 180.0 & 186.0 & 180.0 & 179.9 \\
\hline H3-C3-C4-C9 & 180.0 & 180.0 & 179.9 & 180.0 & -180.0 & 180.0 & 179.9 \\
\hline $\mathrm{O} 16-\mathrm{C} 1-\mathrm{C} 10-\mathrm{C} 11$ & 179.9 & -179.9 & 179.7 & 180.0 & 180.0 & 179.9 & 179.9 \\
\hline $\mathrm{O} 16-\mathrm{C} 1-\mathrm{C} 10-\mathrm{C} 15$ & 0.0 & 0.0 & -0.3 & 0.0 & 0.0 & 0.0 & -0.0 \\
\hline C1-C10-C11-H11 & 0.0 & 0.0 & -0.0 & 0.0 & 0.0 & 0.0 & -0.0 \\
\hline $\mathrm{C} 12-\mathrm{C} 13-\mathrm{O} 17-\mathrm{C} 18$ & 179.9 & 179.9 & 0.0 & 179.9 & 179.9 & 179.9 & 0.0 \\
\hline C14-C13-O17-C18 & 0.0 & 0.0 & -179.9 & -0.0 & 0.0 & 0.0 & -179.9 \\
\hline
\end{tabular}




\begin{tabular}{l|c|c|c|c|c|c|c}
\hline Geometric parameters & $\mathbf{1}$ & $\mathbf{2}$ & $\mathbf{3}$ & $\mathbf{4}$ & $\mathbf{5}$ & $\mathbf{6}$ & $\mathbf{7}$ \\
\hline $\mathrm{C} 13-\mathrm{O} 17-\mathrm{C} 18-\mathrm{H}^{\prime} 18 / \mathrm{H}^{\prime \prime} / \mathrm{H}^{\prime \prime}$ & -130.0 & $61.20 /$ & $61.2 /$ & $61.3 /-$ & $61.3 /$ & $61.1 /$ & $-61.3 /$ \\
& & $179.9 /$ & $179.9 /-$ & $180.0 /-$ & $-179.9 /$ & $180.0 /$ & $61.2 /$ \\
& & 61.2 & 61.2 & 61.3 & -61.3 & 61.3 & 179.9 \\
\hline $\mathrm{C} 5-\mathrm{C} 6-\mathrm{C} 7-\mathrm{X} 19$ & 179.9 & - & 179.9 & -180.0 & 180.0 & 180.0 & -180.0 \\
\hline C6-C7-C19-C20/O20 & - & - & -0.0 & - & - & - & $179.9 /$ \\
& & & & & & -0.0 \\
\hline C14-C10-C1-C2 & 0.0 & 0.0 & -0.4 & -0.0 & -0.0 & 0.0 & -0.0
\end{tabular}

$\mathrm{X}=\mathrm{F}, \mathrm{Cl}, \mathrm{Br}, \mathrm{NO}_{2}$.

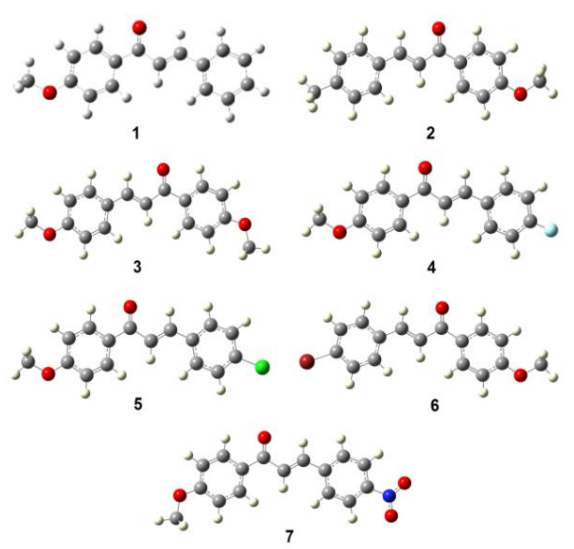

Figure 7. Optimized structures of 1-7.

3.5.2. Natural bond orbital analysis.

Natural bond orbital (NBO) of chalcones 1-7 and the important second-order perturbative estimates of donor-acceptor interactions values are displayed in Table 5. The occupancies and the energies of orbitals involved in primary delocalization are also reported. The interaction between filled and empty Non-bonding orbitals described for the hyper conjugative electron transfer process from the donor (filled) to the acceptor (vacant) orbital and the energy lowering due to this interaction is expressed as E2 [48]. The $\pi$-bonded electrons of $\mathrm{C} 11-\mathrm{C} 12, \mathrm{C} 1-\mathrm{O} 16$, and $\mathrm{C} 14-\mathrm{C} 15$ bonds in compound 5 delocalized onto non-bonding $p$ orbitals (vicinal) available on $\mathrm{C} 10$ and $\mathrm{C} 13$ carbon atoms are found to be very high $(\approx 60,72$ and $57 \mathrm{kcal} \mathrm{mol}^{-1}$ ) and this is primary delocalization of chalcone 3 non-bonding orbital of $\mathrm{C} 1-$ $\mathrm{C} 10$ and $\mathrm{C} 1-\mathrm{C} 2$ bonds delocalization in antibonding orbitals of $\mathrm{C} 2-\mathrm{C} 3$ bond is around 236 and $86 \mathrm{kcal} \mathrm{mol}^{-1}$. The chalcone, 3 methoxy oxygen, donates the electron from aromatic ring to $\alpha, \beta$-unsaturated carbon atoms, the energies are enhanced to $\mathrm{C} 1-\mathrm{C} 2$ atoms. Chalcone 7 lone pair of electrons available on oxygen $\mathrm{O} 20$ and $\mathrm{O} 21$ is delocalized on to the antibonding orbital of $\mathrm{N} 19-\mathrm{O} 20 / \mathrm{O} 21$ bond their delocalization energies are around $\approx 150 \mathrm{kcal} \mathrm{mol}^{-1}$.

Table 5. NBO analysis of 1-7.

\begin{tabular}{l|l|l|l|l|l|l|l|c} 
Donor NBO & Acceptor NBO & $\mathbf{1}$ & $\mathbf{2}$ & $\mathbf{3}$ & $\mathbf{4}$ & $\mathbf{5}$ & $\mathbf{6}$ & $\mathbf{7}$ \\
\hline $\mathrm{BD}(2) \mathrm{C} 2-\mathrm{C} 3$ & $\mathrm{BD}(2) \mathrm{C} 1-\mathrm{O} 16$ & 19.23 & 19.20 & 20.96 & 19.80 & 19.76 & 20.30 & 18.54 \\
\hline $\mathrm{BD}(2) \mathrm{C} 2-\mathrm{C} 3$ & $\mathrm{BD}(2) \mathrm{C} 9-\mathrm{C} 8$ & 12.03 & 12.41 & 12.71 & 12.06 & 12.10 & 11.68 & 13.03 \\
\hline $\mathrm{BD}(2) \mathrm{C} 9-\mathrm{C} 8$ & $\mathrm{BD}(2) \mathrm{C} 2-\mathrm{C} 3$ & 16.21 & 16.25 & 16.37 & 16.52 & 16.45 & 16.60 & 14.99 \\
\hline $\mathrm{BD}(2) \mathrm{C} 9-\mathrm{C} 8$ & $\mathrm{BD}(2) \mathrm{C} 4-\mathrm{C} 5$ & 19.81 & 19.31 & 19.18 & 19.83 & 19.69 & 11.15 & 17.48 \\
\hline $\mathrm{BD}(2) \mathrm{C} 9-\mathrm{C} 8$ & $\mathrm{BD}(2) \mathrm{C} 6-\mathrm{C} 7$ & 22.11 & 22.00 & 22.51 & 22.36 & 22.31 & 19.51 & 23.51 \\
\hline $\mathrm{BD}(2) \mathrm{C} 4-\mathrm{C} 5$ & $\mathrm{BD}(2) \mathrm{C} 9-\mathrm{C} 8$ & 17.80 & 18.05 & 18.31 & 18.30 & 18.51 & 17.05 & 20.55 \\
\hline $\mathrm{BD}(2) \mathrm{C} 4-\mathrm{C} 5$ & $\mathrm{BD}(2) \mathrm{C} 6-\mathrm{C} 7$ & 18.81 & 18.51 & 18.59 & 20.90 & 20.61 & 22.76 & 19.44 \\
\hline $\mathrm{BD}(2) \mathrm{C} 6-\mathrm{C} 7$ & $\mathrm{BD}(2) \mathrm{C} 9-\mathrm{C} 8$ & 18.95 & 19.31 & 19.56 & 18.78 & 18.65 & 21.31 & 17.79 \\
\hline $\mathrm{BD}(2) \mathrm{C} 6-\mathrm{C} 7$ & $\mathrm{BD}(2) \mathrm{C} 4-\mathrm{C} 5$ & 17.61 & 17.75 & 17.51 & 17.87 & 18.50 & 17.79 & 21.43 \\
\hline $\mathrm{BD}(2) \mathrm{C} 10-\mathrm{C} 15$ & $\mathrm{BD}(2) \mathrm{C} 1-\mathrm{O} 18$ & 20.07 & 20.17 & 20.36 & 20.49 & 20.52 & 20.83 & 20.99 \\
\hline $\mathrm{BD}(2) \mathrm{C} 10-\mathrm{C} 15$ & $\mathrm{BD}(2) \mathrm{C} 11-\mathrm{C} 12$ & 22.46 & 22.50 & 22.27 & 22.39 & 22.71 & 23.10 & 22.95 \\
\hline $\mathrm{BD}(2) \mathrm{C} 10-\mathrm{C} 15$ & $\mathrm{BD}(2) \mathrm{C} 13-\mathrm{C} 14$ & 17.46 & 17.74 & 17.86 & 17.52 & 17.48 & 17.56 & 17.39 \\
\hline $\mathrm{BD}(2) \mathrm{C} 11-\mathrm{C} 12$ & $\mathrm{BD}(2) \mathrm{C} 10-\mathrm{C} 15$ & 15.21 & 15.35 & 15.43 & 15.61 & 15.66 & 15.52 & 15.49 \\
\hline $\mathrm{BD}(2) \mathrm{C} 11-\mathrm{C} 12$ & $\mathrm{BD}(2) \mathrm{C} 13-\mathrm{C} 14$ & 22.11 & 22.31 & 22.61 & 22.81 & 22.15 & 22.36 & 22.39 \\
$\mathrm{https} / / /$ biointerfaceresearch.com/ & & & & & & & 7166
\end{tabular}




\begin{tabular}{|c|c|c|c|c|c|c|c|c|}
\hline Donor NBO & Acceptor NBO & 1 & 2 & 3 & 4 & 5 & 6 & 7 \\
\hline $\mathrm{BD}(2) \mathrm{C} 13-\mathrm{C} 14$ & $\mathrm{BD}(2) \mathrm{C} 10-\mathrm{C} 15$ & 24.96 & 25.07 & 25.17 & 25.41 & 25.59 & 24.76 & 25.25 \\
\hline $\mathrm{BD}(2) \mathrm{C} 13-\mathrm{C} 14$ & $\mathrm{BD}(2) \mathrm{C} 11-\mathrm{C} 12$ & 14.86 & 17.97 & 15.03 & 15.09 & 15.36 & 15.58 & 15.16 \\
\hline $\mathrm{LP}(2) \mathrm{O} 18$ & $\mathrm{BD} *(1) \mathrm{C} 1-\mathrm{C} 2$ & 18.96 & 18.42 & 18.71 & 18.96 & 19.23 & 19.47 & 19.39 \\
\hline $\mathrm{LP}(2) \mathrm{O} 18$ & $\mathrm{BD} *(1) \mathrm{C} 1-\mathrm{C} 10$ & 18.83 & 18.33 & 14.44 & 18.57 & 19.02 & 19.44 & 18.31 \\
\hline $\mathrm{LP}(2) \mathrm{O} 16$ & $\mathrm{BD}^{*}(2) \mathrm{C} 13-\mathrm{C} 14$ & 32.02 & 31.83 & 31.96 & 32.01 & 32.21 & 32.44 & 31.91 \\
\hline LP(3)F19 & BD*(1)C6-C7 & - & - & - & 20.16 & - & - & - \\
\hline $\mathrm{BD}(2) \mathrm{C} 11-\mathrm{C} 12$ & $\mathrm{LP}(1) \mathrm{C} 10$ & 37.86 & 37.44 & 37.96 & 38.08 & 38.26 & 38.00 & 38.16 \\
\hline $\mathrm{BD}(2) \mathrm{C} 1-\mathrm{O} 16$ & $\mathrm{BD} *(2) \mathrm{C} 2-\mathrm{C} 3$ & 13.76 & 13.16 & 13.26 & 14.11 & 14.21 & 13.96 & 13.36 \\
\hline $\mathrm{BD}(2) \mathrm{C} 14-\mathrm{C} 15$ & $\mathrm{LP}(1) \mathrm{C} 10$ & - & - & - & - & 37.74 & - & - \\
\hline $\mathrm{LP}(1) \mathrm{C} 10$ & $\mathrm{BD} *(12) \mathrm{C} 1-\mathrm{O} 16$ & - & - & - & - & 60.37 & - & - \\
\hline $\mathrm{LP}(1) \mathrm{C} 10$ & $\mathrm{BD} *(2) \mathrm{C} 11-\mathrm{C} 12$ & - & - & - & - & 72.70 & - & - \\
\hline $\mathrm{LP}(1) \mathrm{C} 13$ & $\mathrm{BD} *(2) \mathrm{C} 11-\mathrm{C} 12$ & - & - & - & - & 52.84 & - & - \\
\hline $\mathrm{LP}(2) \mathrm{O} 16$ & $\mathrm{LP} *(1) \mathrm{C} 13$ & - & - & - & - & 57.20 & - & - \\
\hline $\mathrm{BD}(2) \mathrm{C} 11-\mathrm{C} 12$ & $\mathrm{LP} *(1) \mathrm{C} 13$ & - & - & - & - & 55.05 & - & - \\
\hline $\mathrm{BD}(2) \mathrm{C} 14-\mathrm{C} 15$ & $\mathrm{LP} *(1) \mathrm{C} 13$ & - & - & - & - & 62.08 & - & - \\
\hline $\mathrm{LP}(3) \mathrm{Cl} 19$ & $\mathrm{BD} *(2) \mathrm{C} 7-\mathrm{C} 8$ & - & - & - & - & 21.32 & - & - \\
\hline $\mathrm{BD}(2) \mathrm{N} 19-\mathrm{O} 20$ & BD*(1)C15-H15 & - & - & - & - & - & - & 17.16 \\
\hline $\mathrm{BD}(2) \mathrm{N} 19-\mathrm{O} 20$ & $\mathrm{BD} *(1) \mathrm{O} 17-\mathrm{C} 18$ & - & - & - & - & - & - & 25.64 \\
\hline $\mathrm{BD}(1) \mathrm{N} 19-\mathrm{O} 20$ & $\mathrm{BD}^{*}(2) \mathrm{N} 19-\mathrm{O} 20$ & - & - & - & - & - & - & 13.27 \\
\hline $\mathrm{LP}(2) \mathrm{O} 20$ & BD*(1)C7-N19 & - & - & - & - & - & - & 12.30 \\
\hline $\mathrm{LP}(2) \mathrm{O} 21$ & BD*(1)C7-N19 & - & - & - & - & - & - & 11.92 \\
\hline $\mathrm{LP}(3) \mathrm{O} 21$ & BD*(1)O16-C17 & - & - & - & - & - & - & 368.11 \\
\hline
\end{tabular}

\subsubsection{Atoms-in-molecules analysis.}

Atoms-in-molecules (AIM) electron density topological analysis carried out for chalcone 1-7. Around 30 bond critical points (BCPs) with a $(3,-1)$ topology between the atoms connected by a covalent bond (Table 6). The observed negative values for the Laplacian are indicated to the interaction of covalent or polarized bonds and are characterized by large $\rho_{\mathrm{b}}$ values. Besides these one bonds, critical points are observed between the atoms $\mathrm{C} 1$ and $\mathrm{O} 16$ (carbonyl group) atom. The values of $\rho_{\mathrm{BCP}}$ at the $\mathrm{BCP}$ are found to be 4.23 and $\nabla^{2} \rho_{\mathrm{BCP}}$. Values are found to be 2.90 . The positive magnitude of $\nabla^{2} \rho_{\mathrm{BCP}}$ indicates the highly ionic nature of the carbonyl group in chalcones 1-7. In other bonds, is ionic nature of the carbonyl (C1-O16) group is confirmed by the positive Laplacian density at the critical bond point and high $\rho_{\mathrm{BCP}}$ values $[47,48]$. In chalcones 4-7, another (1-3) BCP was also located between C7-X19 atoms. The Laplacian of the density at the BCP is attained positive and $\nabla^{2} \rho$ highly positive indicating the ionic nature of the $\mathrm{C} 7-\mathrm{X} 19$ bonds also.

3.5.4. Energies, dipole moments and polarizabilities.

HOMO-LUMO and dipole moment values for the chalcones 1-7 are listed in Table 7. HOMO-LUMO figures are displayed in Fig. 8. In the HOMO and LUMO orbitals, the introduction of electron-withdrawing groups for nitro, bromo, chloro, fluoro in para substitution for aromatic ring decreases the energies, and electron releasing methyl substituent and parent chalcone increases the energies of both HOMO and LUMO orbitals. Especially, compound 7 had the smallest HOMO-LUMO gap $(\Delta \mathrm{E}=0.127 \mathrm{eV})$, which signifies rapid electron and radical transfer between HOMO and LUMO orbital [49]. This could be one of the reasons that compound $\mathbf{7}$ showed good free radical scavenging activity. This finding reveals a close relationship to the electron density of compound $\mathbf{7}$ based on the resonance effect. The dipole moment is increased due to the presence of electron electron-withdrawing substituents (nitro, bromo, chloro, and fluoro) in the para position of the phenyl ring $\mathrm{C}_{2}=\mathrm{C}_{3}$ - and decrease due to the presence of electron releasing methyl substituent. The order of dipole moments is 1 $>2>4>5>6>3>7$. 
Table 6. AIM parameters for 1-7.

Bond parameters

\begin{tabular}{|c|c|c|c|c|c|c|c|c|c|c|c|c|c|c|c|c|c|c|c|c|c|}
\hline \multirow{3}{*}{ Bond } & \multirow{2}{*}{\multicolumn{7}{|c|}{$\rho \times 10^{1}$}} & \multirow{2}{*}{\multicolumn{7}{|c|}{$\nabla^{2} \rho \times 10^{1}$}} & \multirow{2}{*}{\multicolumn{7}{|c|}{$\varepsilon \times 10^{1}$}} \\
\hline & & & & & & & & & & & & & & & & & & & & & \\
\hline & 1 & 2 & 3 & 4 & 5 & 6 & 7 & 1 & 2 & 3 & 4 & 5 & 6 & 7 & 1 & 2 & 3 & 4 & 5 & 6 & 7 \\
\hline C1-O6 & 4.23 & 4.23 & 4.23 & 4.23 & 4.23 & 4.23 & 4.23 & 2.90 & 2.91 & 2.93 & 2.94 & 2.94 & 2.94 & 2.94 & 0.60 & 0.59 & 0.59 & 0.59 & 0.59 & 0.59 & 0.60 \\
\hline $\mathrm{C} 1-\mathrm{C} 2$ & 3.50 & 3.49 & 3.49 & 3.49 & 3.49 & 3.49 & 3.50 & -8.49 & -8.45 & -8.45 & 8.46 & -8.46 & -8.46 & -8.47 & 1.08 & 1.08 & 1.08 & 1.09 & 1.09 & 1.09 & 1.07 \\
\hline $\mathrm{C} 2-\mathrm{C} 3$ & 3.39 & 3.38 & 3.38 & 3.37 & 3.37 & 3.37 & 3.36 & -7.41 & -7.39 & -7.39 & -7.37 & -7.37 & -7.38 & -7.40 & 1.02 & 1.02 & 1.02 & 1.02 & 1.02 & 1.02 & 1.01 \\
\hline C3-C4 & 3.03 & 3.03 & 3.03 & 3.04 & 3.04 & 3.04 & 3.05 & -9.71 & -9.72 & -9.72 & -9.74 & -9.74 & -9.74 & -9.89 & 0.80 & 0.80 & 0.80 & 0.81 & 0.82 & 0.82 & 0.87 \\
\hline C4-C5 & 3.14 & 3.14 & 3.14 & 3.14 & 3.15 & 3.15 & 3.13 & -8.67 & -8.71 & -8.71 & -8.62 & -8.62 & -8.62 & -8.67 & 2.18 & 2.21 & 2.23 & 2.23 & 2.23 & 2.23 & 2.10 \\
\hline C5-C6 & 3.13 & 3.14 & 3.12 & 3.13 & 3.13 & 3.13 & 3.15 & -8.59 & -8.59 & -8.50 & -8.53 & -8.53 & -8.53 & -8.65 & 2.11 & 2.14 & 2.14 & 2.20 & 2.20 & 2.20 & 2.08 \\
\hline C6-C7 & 3.13 & 3.11 & 3.20 & 3.21 & 3.21 & 3.22 & 3.15 & -8.54 & -8.40 & -8.40 & -9.10 & -9.10 & -9.11 & -8.73 & 2.11 & 2.15 & 2.15 & 2.68 & 2.68 & 2.68 & 2.18 \\
\hline C7-X19 & - & 2.55 & 2.52 & 2.56 & 2.57 & 2.57 & 2.64 & - & -6.50 & -6.50 & 1.41 & 1.40 & 1.41 & 7.48 & - & 0.31 & 0.31 & 0.71 & 0.71 & 0.72 & 1.17 \\
\hline C7-C8 & 3.17 & 3.16 & 3.16 & 3.16 & 3.16 & 3.16 & 3.16 & -8.83 & -8.83 & -8.81 & -8.81 & -8.82 & -8.82 & -8.80 & 2.17 & 2.16 & 2.16 & 2.16 & 2.16 & 2.16 & 2.13 \\
\hline C8-C9 & 3.09 & 3.09 & 3.09 & 3.09 & 3.09 & 3.09 & 3.08 & -8.43 & -8.43 & -8.42 & -8.41 & -8.41 & -8.41 & -8.40 & 2.37 & 2.39 & 2.39 & 2.37 & 2.37 & 2.36 & 2.32 \\
\hline $\mathrm{C} 1-\mathrm{C} 10$ & 2.72 & 2.71 & 2.71 & 2.71 & 2.71 & 2.71 & 2.70 & -6.81 & -6.78 & -6.77 & -6.75 & -6.75 & -6.75 & -6.75 & 1.09 & 1.07 & 1.07 & 1.06 & 1.06 & 1.06 & 1.05 \\
\hline $\mathrm{C} 11-\mathrm{C} 12$ & 3.08 & 3.08 & 3.08 & 3.07 & 3.07 & 3.07 & 3.06 & -8.31 & -8.31 & -8.31 & -8.30 & -8.30 & -8.30 & -8.28 & 1.91 & 1.92 & 1.92 & 1.90 & 1.90 & 1.90 & 1.89 \\
\hline $\mathrm{C} 12-\mathrm{C} 13$ & 3.06 & 3.06 & 3.06 & 3.05 & 3.05 & 3.05 & 3.04 & -8.30 & -8.29 & -8.29 & -8.28 & -8.28 & -8.28 & -8.27 & 1.89 & 1.89 & 1.89 & 1.89 & 1.89 & 1.90 & 1.91 \\
\hline C13-O16 & 2.87 & 2.87 & 2.88 & 2.88 & 2.88 & 2.89 & 2.90 & -2.89 & -2.88 & -2.88 & -2.87 & -2.87 & -28.7 & -2.83 & 0.10 & 0.09 & 0.09 & 0.10 & 0.10 & 0.10 & 0.13 \\
\hline O16-C17 & 2.31 & 2.32 & 2.32 & 2.31 & 2.31 & 2.31 & 2.31 & -3.58 & -3.58 & -3.60 & -3.59 & -3.59 & -3.59 & -3.54 & 0.26 & 0.27 & 0.27 & 0.30 & 0.30 & 0.30 & 0.27 \\
\hline C13-C14 & 3.11 & 3.11 & 3.11 & 3.12 & 3.12 & 3.12 & 3.12 & -8.43 & -8.43 & -8.43 & -8.45 & -8.45 & -8.46 & -8.49 & 1.92 & 1.92 & 1.92 & 1.92 & 1.92 & 1.92 & 1.93 \\
\hline C14-C15 & 3.15 & 3.14 & 3.15 & 3.15 & 3.15 & 3.15 & 3.16 & -8.58 & -8.56 & -8.56 & -8.57 & -8.57 & -8.57 & -8.62 & 2.23 & 2.24 & 2.24 & 2.24 & 2.24 & 2.24 & 2.25 \\
\hline C15-C16 & 3.10 & 3.10 & 3.10 & 3.12 & 3.12 & 3.12 & 3.12 & -8.40 & -8.40 & -8.40 & -8.41 & -8.41 & -8.41 & -8.45 & 1.89 & 1.89 & 1.89 & 1.89 & 1.89 & 1.89 & 1.90 \\
\hline
\end{tabular}
Ring parameters

\begin{tabular}{|c|c|c|c|c|c|c|c|c|}
\hline \multirow{3}{*}{\multicolumn{2}{|c|}{ Ring }} & \\
\hline & & \multicolumn{7}{|c|}{$\rho \times 10^{2}$} \\
\hline & & 1 & 2 & 3 & 4 & 5 & 6 & 7 \\
\hline 1 & C4-C5-C6-C7-C8-C9 & 1.99 & 1.99 & 1.99 & 1.99 & 1.99 & 1.99 & 2.00 \\
\hline 2 & $\mathrm{C} 10-\mathrm{C} 11-\mathrm{C} 12-\mathrm{C} 13-\mathrm{C} 14-\mathrm{C} 5$ & 2.02 & 2.00 & 2.00 & 2.00 & 2.00 & 2.00 & 2.04 \\
\hline
\end{tabular}

$\mathrm{X}=\mathrm{F}, \mathrm{Cl}, \mathrm{Br}, \mathrm{NO}_{2}$.

Table 7. HOMO-LUMO energies (eV) and dipole moments $\mu(D)$ of 1-7.

\begin{tabular}{|c|c|c|c|c|c|c|c|}
\hline \multirow{2}{*}{ Compds. } & \multicolumn{3}{|c|}{ HOMO-LUMO energies } & \multicolumn{4}{|c|}{ Dipole moments } \\
\hline & HOMO & LUMO & $\Delta E$ & $\mu_{\mathrm{x}}$ & $\mu_{\mathrm{y}}$ & $\mu_{\mathrm{z}}$ & $\mu_{\text {tot }}$ \\
\hline 1 & -0.253 & -0.095 & 0.158 & 1.909 & 2.964 & 0.201 & 3.531 \\
\hline 2 & -0.245 & -0.090 & 0.155 & 0.077 & -2.508 & 0.017 & 2.510 \\
\hline 3 & -0.210 & -0.065 & 0.146 & 0.278 & -3.849 & 0.005 & 3.859 \\
\hline 4 & -0.233 & -0.084 & 0.149 & 2.125 & -1.628 & 0.000 & 2.677 \\
\hline 5 & -0.240 & -0.093 & 0.147 & 2.688 & -1.666 & 0.000 & 3.163 \\
\hline 6 & -0.239 & -0.093 & 0.146 & 1.517 & -0.746 & 0.000 & 1.691 \\
\hline 7 & -0.236 & -0.106 & 0.130 & -6.909 & -0.547 & 0.000 & 6.931 \\
\hline
\end{tabular}




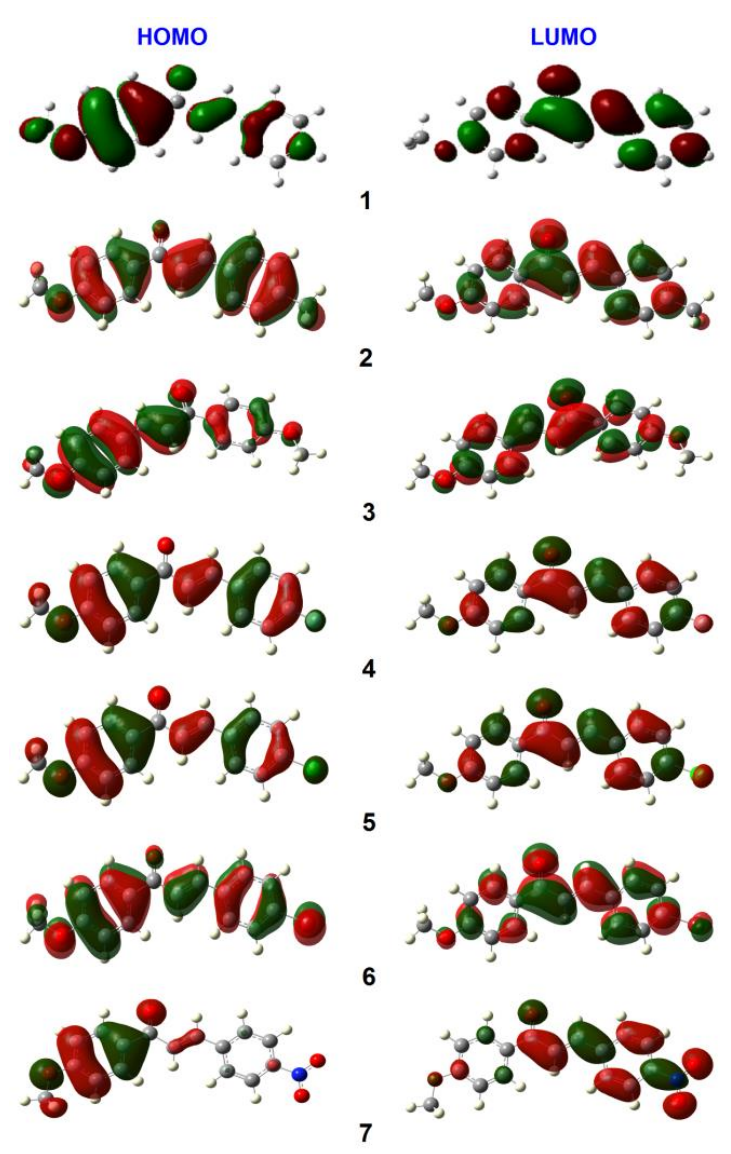

Figure 8. HOMO-LUMO pictures of 1-7.

HOMO and LUMO orbitals are derived from pz orbitals of carbon, nitrogen, oxygen, and halogens $\left(\mathrm{X}=\mathrm{F}, \mathrm{Cl}, \mathrm{Br}\right.$, and $\left.\mathrm{NO}_{2}\right)$ atoms except $p z$ orbitals of methoxy oxygen $\mathrm{O}-17$ in 1-7. Compound 7, the $p z$ orbital of $\alpha, \beta$-unsaturated carbon $\mathrm{C}-4$ linked with nitro substituted benzene ring does not participate to HOMO orbital, the same way $p z$ orbital of LUMO orbital of a carbonyl group linked methoxy benzene ring also not involved LUMO orbital. In 2, 4, 5, and 6, all the $p z$ orbitals are contributed to HOMO orbital else to $\mathrm{C}-1$ atom, and the LUMO $p z$ orbital of $\mathrm{C}-6, \mathrm{C}-10, \mathrm{C}-12, \mathrm{C}-14$, and $\mathrm{O}-17$ orbital do not participate to LUMO orbital. The methoxy substituted chalcone $3, \mathrm{C}-15, \mathrm{C}-12, \mathrm{O}-17$, and $\mathrm{C}-18$ atoms are not sharing $p z$ orbital of HOMO. The LUMO orbitals of C-12, C-14, C-6, C-18, O-17, C-18, O-19, and C-20 are not involved in the LUMO orbitals.

The polarizability $\left(\alpha_{0}\right)$ and first-order hyperpolarizability $\left(\beta_{\text {tot }}\right)$ are related directly to the non-linear optical efficiency of structures. The calculated values of $\alpha_{0}, \beta_{\text {tot }}$ by the finite field approach are given in Table 8. The calculated values of $\alpha_{\mathrm{ij}}, \beta_{\mathrm{ijk}}$ are converted into electrostatic units from atomic units $\left(\alpha\right.$ : 1 a.u $=0.1482 \times 10^{-24} \mathrm{esu} ; \beta$ : $\left.1 \mathrm{a} . \mathrm{u}=8.6393 \times 10^{-33} \mathrm{esu}\right)$. The electron-withdrawing substituent of $\mathrm{NO}_{2}, \mathrm{Cl}$, and $\mathrm{F}$ moieties of chalcones are increased the $\beta_{\text {tot }}$ values. The NLO character increasing according to the following order: $7>5>4>1$ $>3>2>6$.

Table 8. Polarizabilities and hyperpolarizabilities for 1-7.

\begin{tabular}{c|c|c|c|c|c|c|c} 
& $\mathbf{1}$ & $\mathbf{2}$ & $\mathbf{3}$ & $\mathbf{4}$ & $\mathbf{5}$ & $\mathbf{6}$ & $\mathbf{7}$ \\
\hline$\alpha_{\mathrm{xx}}$ & 380.601 & -69.0903 & -74.3582 & -89.8280 & -95.0822 & -95.9070 & -140.5985 \\
\hline$\alpha_{\mathrm{xy}}$ & -0.028 & 4.2553 & -18.4817 & 2.9736 & 3.8946 & -5.6540 & 26.9686 \\
\hline$\alpha_{\mathrm{yy}}$ & 66.887 & -114.3261 & -113.1848 & -111.3271 & -119.2618 & -125.0768 & 10.0652 \\
\hline$\alpha_{\mathrm{xz}}$ & -91.575 & -0.1728 & -0.0154 & -0.0044 & 0.0000 & 0.0001 & -0.0035 \\
\hline$\alpha_{\mathrm{yz}}$ & -0.001 & -0.0379 & -0.0181 & 0.0022 & 0.0000 & 0.0000 & -0.0014
\end{tabular}




\begin{tabular}{c|c|c|c|c|c|c|c}
\hline & $\mathbf{1}$ & $\mathbf{2}$ & $\mathbf{3}$ & $\mathbf{4}$ & $\mathbf{5}$ & $\mathbf{6}$ & $\mathbf{7}$ \\
\hline$\alpha_{\mathrm{zz}}$ & 238.479 & -118.5810 & -119.8505 & -115.6792 & -123.8500 & -129.7882 & 4.4855 \\
\hline$\langle\alpha>$ (a.u) & 224.170 & 100.6658 & 102.4644 & 105.4114 & 84.0647 & 116.9240 & 42.0159 \\
\hline $10^{24} \times \alpha_{\text {tot }}(\mathrm{esu})$ & 33.222 & 14.9187 & 15.1852 & 15.6220 & 12.4584 & 17.3281 & 6.2267 \\
\hline$\beta_{\mathrm{xxx}}$ & 173.269 & 120.4287 & -115.0879 & -289.1997 & -334.6637 & 214.7746 & -441.9497 \\
\hline$\beta_{\mathrm{xxy}}$ & -3.425 & 20.7522 & -38.0341 & 76.6685 & 56.4469 & 23.7860 & 1.3231 \\
\hline$\beta_{\mathrm{xyy}}$ & 19.204 & -19.4936 & 31.6132 & 31.6056 & 0.984 & -94.0877 & -38.6199 \\
\hline$\beta_{\mathrm{yyy}}$ & -1.949 & -24.6910 & -35.4373 & -33.2391 & -26.0961 & -43.2497 & -31.0270 \\
\hline$\beta_{\mathrm{xxz}}$ & -37.537 & 0.8559 & 0.3678 & 0.0298 & 0.0004 & 0.0006 & 0.0345 \\
\hline$\beta_{\mathrm{xyz}}$ & 1.639 & 0.2615 & -0.2309 & 0.0077 & 0.0002 & 0.0001 & 0.0042 \\
\hline$\beta_{\mathrm{yyz}}$ & -45.132 & -0.3487 & -0.0181 & 0.0023 & 0.0001 & 0.0000 & -0.0019 \\
\hline$\beta_{\mathrm{xzz}}$ & -137.565 & 15.9789 & 108975 & 2.0775 & -6.0056 & -55.1076 & 16.0328 \\
\hline$\beta_{\mathrm{yzz}}$ & -0.729 & 0.7669 & -0.9980 & -1.4814 & -0.3591 & -7.9544 & -04.6646 \\
\hline$\beta_{\mathrm{zzz}}$ & 247.633 & 0.4710 & 0.0022 & 0.0110 & 0.0001 & 0.0001 & -0.0003 \\
\hline$\beta_{\text {tot }}(\mathrm{a} . \mathrm{u})$ & 173.939 & 117.3502 & 142.5722 & 258.9286 & 312.0655 & 71.0802 & 465.3064 \\
\hline $10^{33} \times \beta_{\text {tot }}(\mathrm{esu})$ & 1502.710 & 1013.8233 & 1231.7240 & 2236.9618 & 2696.0271 & 614.0833 & 40.2415
\end{tabular}

3.5.5. Molecular electrostatic potential surfaces.

The MEP surface has been plotted for chalcones, and the representative diagram is given in Fig. 9. The red color region has appeared electronegative oxygen $\mathrm{O}-1$ and $\mathrm{O}-17$ in chalcones 1-7. The red-colored locations are polarized for the electrophilic attack [49], while the blue indicates a greater positive region and the maximum green color in the MEP surfaces regarding a potential halfway between the two extremes [red and blue] regions in chalcones 17.
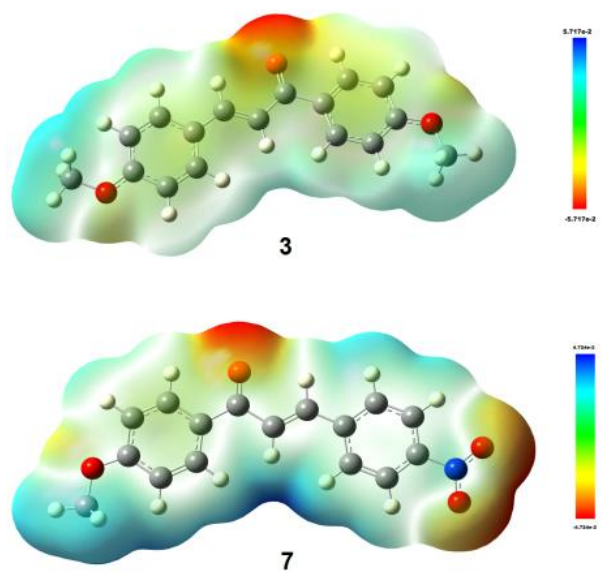

Figure 9. MEP diagrams of 3 and 7.

\subsection{Spectral properties.}

The computational ${ }^{1} \mathrm{H}$ and ${ }^{13} \mathrm{C}$ NMR chemical shifts values are compared. Normally theoretical ${ }^{1} \mathrm{H}$ chemical shifts values are higher when compared to experimental values, and some proton values have largely deviated from experimental values. The optimized structure, proton positions are three-dimensionally calculated in computation in ${ }^{1} \mathrm{H}$ chemical shifts. The ${ }^{13} \mathrm{C}$ chemical shifts are closer to the theoretical values. The correlation between experimental and theoretical ${ }^{13} \mathrm{C}$ chemical shifts is shown in Fig. 10.

\subsection{Charges.}

Charges derived from NBO calculations are listed in Table 9, along with the Mulliken charges. From Table 9, it is inferred that all hydrogen and $s p^{2}$ carbons of some of the carbon atoms attain positive charges $(\mathrm{C}-3, \mathrm{C}-6, \mathrm{C}-9, \mathrm{C}-10, \mathrm{C}-14$, and $\mathrm{C}-18)$ while the remaining 
carbon atoms attain negative charges. The charge sharing the molecule is a significant influence of the atoms possess negative and positive.
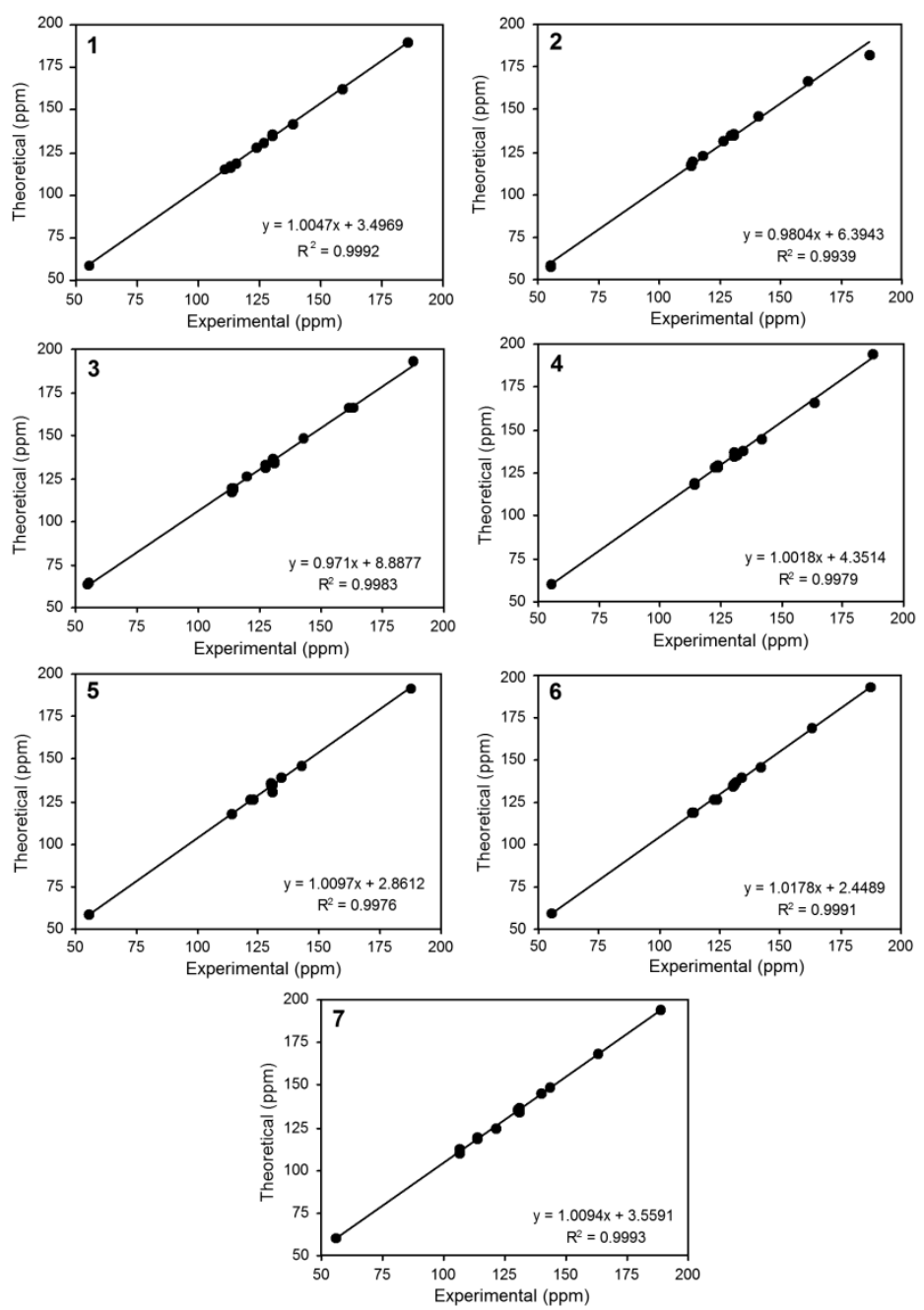

Figure 10. Correlation between the experimental and theoretical ${ }^{13} \mathrm{C}$ chemical shifts for $1-7$.

It is observed that the negative charge on $\mathrm{O} 17$ and $\mathrm{O} 19$, resulting in polarizing the positive charge on the adjacent atoms of $\mathrm{C}-3, \mathrm{C}-6, \mathrm{C}-9, \mathrm{C}-10, \mathrm{C}-14$, and $\mathrm{C}-18$. In this, positive carbon atoms act as an electrophilic center in the molecule. The remaining carbon atoms are acting nucleophilic center the atoms. The charges predicted from NBO calculations are slightly higher than those of Mulliken charges on all carbons except those on $\mathrm{C}-3, \mathrm{C}-6, \mathrm{C}-$ 9, C-10, C-14, and C-18.

\subsection{Antimicrobial studies.}

The antimicrobial activity of chalcones 1-7 was studied difference concentration of the disc diffusion method using the Kirby-Bauer method [50]. The bacterial strains viz., Staphylococcus aureus, Escherichia coli, and fungal strains viz., Candida albicans, Aspergillus flavus were used for this study. The zone of inhibition ( $\mathrm{mm}$ ) of chalcones 1-7 is tested for both bacterial and fungal strains and values [51,52] listed in Table 10. The selected photographs are displayed in Fig.11. The Ethanol solvent was used to control the Gentamicin and Fluconazole standards for bacterial and fungal strains, respectively. The compounds 2, 5, and 6 demonstrated higher activity against antibacterial strains E. coli and S. aureus. 
Table 9. Charges (Mulliken and Natural) of 1-7.

\begin{tabular}{|c|c|c|c|c|c|c|c|c|c|c|c|c|c|c|}
\hline \multirow{3}{*}{ Atoms } & \multirow{2}{*}{\multicolumn{2}{|c|}{1}} & \multirow{2}{*}{\multicolumn{2}{|c|}{2}} & \multirow{2}{*}{\multicolumn{2}{|c|}{3}} & \multirow{2}{*}{\multicolumn{2}{|c|}{4}} & \multirow{2}{*}{\multicolumn{2}{|c|}{5}} & \multirow{2}{*}{\multicolumn{2}{|c|}{6}} & \multirow{2}{*}{\multicolumn{2}{|c|}{7}} \\
\hline & & & & & & & & & & & & & & \\
\hline & MC & $\mathrm{NC}$ & MC & $\mathrm{NC}$ & MC & NC & MC & NC & MC & NC & MC & NC & MC & $\mathrm{NC}$ \\
\hline $\mathrm{C}-1$ & -0.6327 & 0.5797 & -0.6471 & 0.5182 & -0.6682 & 0.5048 & 0.3736 & 0.5216 & -0.8352 & 0.5033 & -0.8194 & 0.5034 & -0.8357 & -0.5027 \\
\hline $\mathrm{C}-2$ & -0.1133 & -0.2934 & -0.1263 & -0.2851 & -0.0596 & -0.3011 & -0.0637 & -0.3099 & 0.1165 & -0.2707 & 0.0124 & -0.2703 & 0.1974 & -0.2455 \\
\hline $\mathrm{C}-3$ & 0.2674 & -0.0836 & 0.2741 & -0.0852 & 0.4348 & -0.0779 & 0.2094 & -0.1446 & 0.2921 & -0.0932 & 0.8019 & -0.0927 & 0.3892 & -0.1081 \\
\hline $\mathrm{C}-4$ & -0.4531 & -0.1554 & -0.4623 & -0.1594 & -0.4456 & -0.1500 & -0.0165 & -0.1879 & -0.0509 & -0.1407 & -0.6394 & -0.1412 & -0.6466 & -0.1549 \\
\hline $\mathrm{C}-5$ & -0.2296 & -0.1999 & -0.2305 & -0.1983 & 0.1417 & -0.2757 & -0.0334 & -0.3026 & -0.1494 & -0.2255 & -0.2424 & -0.2266 & 0.2705 & -0.1863 \\
\hline $\mathrm{C}-6$ & 0.5787 & -0.3147 & 0.5898 & -0.0128 & -0.7832 & 0.3358 & 0.3585 & 0.4312 & 0.4376 & -0.0278 & 0.1322 & -0.0938 & -0.7019 & 0.0770 \\
\hline $\mathrm{N}-7$ & -0.4323 & -0.2131 & -0.5836 & -0.2037 & -0.1461 & -0.2392 & -0.0302 & -0.3053 & -0.5450 & -0.2295 & -0.1988 & -0.2243 & -0.1930 & -0.1860 \\
\hline $\mathrm{N}-8$ & -0.2137 & -0.1516 & -0.2331 & -0.1546 & -0.0272 & -0.1456 & -0.0265 & -0.1872 & 0.0533 & -0.1356 & 0.0379 & -0.1491 & 0.0784 & -0.1499 \\
\hline $\mathrm{C}-9$ & 0.8313 & -0.1176 & 0.9418 & -0.1065 & 1.1007 & -0.1269 & 0.1287 & -0.1005 & 0.7969 & -0.1098 & 1.0962 & -0.1024 & 1.1834 & -0.0718 \\
\hline $\mathrm{C}-10$ & 1.3934 & -0.1462 & 1.5012 & -0.1575 & 1.4274 & -0.1482 & 0.0378 & -0.1659 & 1.9285 & -0.1528 & 1.8832 & -0.1529 & 1.9716 & -0.1564 \\
\hline $\mathrm{C}-11$ & -0.7931 & -0.1567 & -0.8664 & -0.1597 & -0.7905 & -0.1599 & -0.0253 & -0.1925 & 1.2195 & -0.1588 & -1.1619 & -0.1588 & -1.1944 & -0.1576 \\
\hline $\mathrm{C}-12$ & -0.4000 & -0.2431 & -0.4027 & -0.2444 & -0.3993 & -0.2444 & -0.0310 & -0.2809 & -0.1924 & -0.2435 & -0.2411 & -0.2434 & -0.1801 & -0.2428 \\
\hline $\mathrm{C}-13$ & -0.5321 & -0.5416 & -0.5417 & 0.3465 & -0.5477 & 0.3454 & 0.3630 & 0.3430 & -0.7229 & 0.3485 & -0.6422 & 0.3489 & -0.7533 & 0.3527 \\
\hline $\mathrm{C}-14$ & 0.3361 & -0.2931 & 0.3394 & -0.2901 & 0.3393 & -0.2900 & 0.0458 & -0.3240 & 0.1558 & -0.2891 & 0.1949 & -0.2890 & 0.1299 & -0.2878 \\
\hline $\mathrm{C}-15$ & 0.6931 & -0.1326 & 0.7081 & -0.1344 & 0.6503 & -0.1342 & 0.0108 & -0.1637 & 0.6695 & -0.1317 & 0.6801 & -0.1316 & 0.6718 & -0.1290 \\
\hline $\mathrm{O}-16$ & -0.2416 & 0.5914 & -0.2486 & -0.5903 & -0.2521 & -0.5927 & -0.5026 & -0.5765 & -0.2385 & -0.5857 & -0.2398 & -0.5854 & -0.2307 & -0.5779 \\
\hline $\mathrm{O}-17$ & -1507 & 0.5344 & -0.1520 & -0.5351 & -0.1534 & -0.5757 & -0.5104 & -0.5134 & -0.1508 & -0.5337 & -0.1511 & -0.5336 & -0.1475 & -0.5313 \\
\hline $\mathrm{O}-18$ & 0.1831 & -0.5317 & 0.1811 & -0.2061 & 0.1783 & -0.2059 & 0.2842 & -0.3289 & 0.1759 & -0.2064 & 0.1794 & -0.2063 & 0.1794 & -0.2060 \\
\hline $\mathrm{X}-19$ & - & - & -0.0699 & -0.5913 & -0.1527 & -0.5359 & -0.2955 & -0.3309 & 0.4784 & 0.0096 & -0.1792 & 0.0743 & -0.1865 & 0.4829 \\
\hline $\begin{array}{l}\mathrm{O}-20 / \\
\mathrm{C}-20\end{array}$ & - & - & - & - & 0.1532 & -0.2061 & - & - & - & - & - & - & 0.0009 & -0.3776 \\
\hline O-21 & - & - & - & - & - & - & - & - & - & - & - & - & -0.0027 & -0.3789 \\
\hline
\end{tabular}

$\mathrm{X}=\mathrm{F}, \mathrm{Cl}, \mathrm{Br}, \mathrm{NO}_{2} ; \mathrm{MC}-$ Mulliken charges; $\mathrm{NC}-$ Natural charges

Table 10. Antibacterial and antifungal activities of 1-7.

Antibacterial

\begin{tabular}{|c|c|c|c|c|c|c|c|c|c|c|c|c|c|c|c|c|}
\hline \multirow{5}{*}{ Compds. } & \multicolumn{8}{|c|}{ Antibacterial } & \multicolumn{8}{|c|}{ Antifungal } \\
\hline & \multicolumn{16}{|c|}{ Zone of inhibition (mm) } \\
\hline & \multicolumn{4}{|c|}{ Staphylococcus aureus } & \multicolumn{4}{|c|}{ Escherichia coli } & \multicolumn{4}{|c|}{ Candida albicans } & \multicolumn{4}{|c|}{ Aspergillus flavus } \\
\hline & \multicolumn{3}{|c|}{$\mu \mathrm{g} / \mathrm{mL}$} & \multirow{2}{*}{$\mathrm{G}(18 \mathrm{~mm})$} & \multicolumn{3}{|c|}{$\mu \mathrm{g} / \mathrm{mL}$} & \multirow{2}{*}{$\mathrm{G}(18 \mathrm{~mm})$} & \multicolumn{3}{|c|}{$\mu \mathrm{g} / \mathrm{mL}$} & \multirow{2}{*}{ F $(15 \mathrm{~mm})$} & \multicolumn{3}{|c|}{$\mu \mathrm{g} / \mathrm{mL}$} & \multirow{2}{*}{$F(16 \mathrm{~mm})$} \\
\hline & 10 & 20 & 40 & & 10 & 20 & 40 & & 10 & 20 & 40 & & 10 & 20 & 40 & \\
\hline 1 & 13 & 15 & 22 & 6 & 10 & 12 & 11 & 22 & 11 & 15 & 12 & 14 & 12 & 18 & 20 & 16 \\
\hline 2 & 14 & 17 & 24 & 8 & 15 & 17 & 14 & 19 & 13 & 18 & 13 & 14 & 14 & 20 & 22 & 17 \\
\hline 3 & 8 & 9 & 14 & 7 & 10 & 11 & 12 & 19 & 10 & 9 & 14 & 12 & 21 & 12 & 15 & 14 \\
\hline 4 & - & - & 12 & 23 & 7 & 7 & 11 & 31 & 10 & 12 & 11 & 20 & 9 & 11 & 12 & 21 \\
\hline 5 & 16 & 20 & 16 & - & - & - & 18 & 18 & 12 & 16 & 13 & 17 & 12 & 21 & 22 & 16 \\
\hline 6 & 16 & 16 & 18 & - & 13 & 18 & 17 & 18 & 9 & 13 & 14 & 15 & - & 13 & 15 & 16 \\
\hline 7 & 9 & - & 7 & - & 12 & 7 & 9 & 30 & 20 & 18 & 18 & 20 & 7 & 11 & - & 18 \\
\hline
\end{tabular}

*Activity for the reference compound: for bacteria (1-7) $\mathrm{G}-$ Gentamicin was used as reference; for fungus (1-7) F-Fluconazole was used as reference 


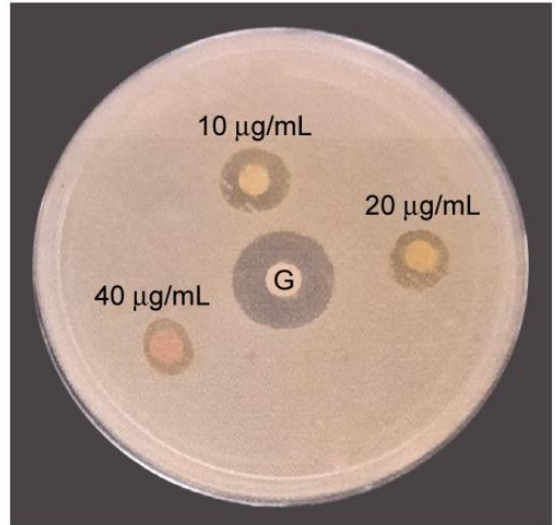

Escherichia coli

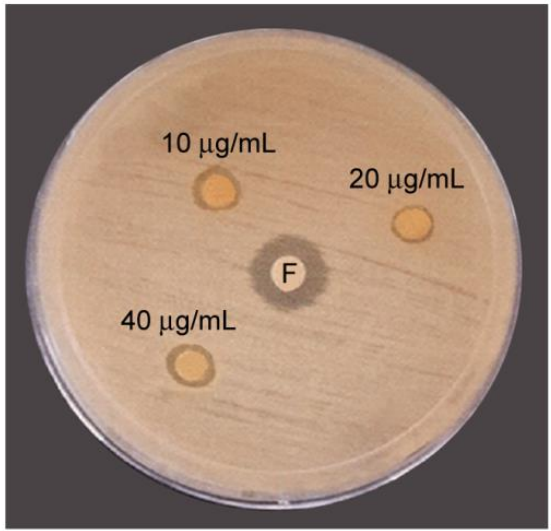

Candida albicans

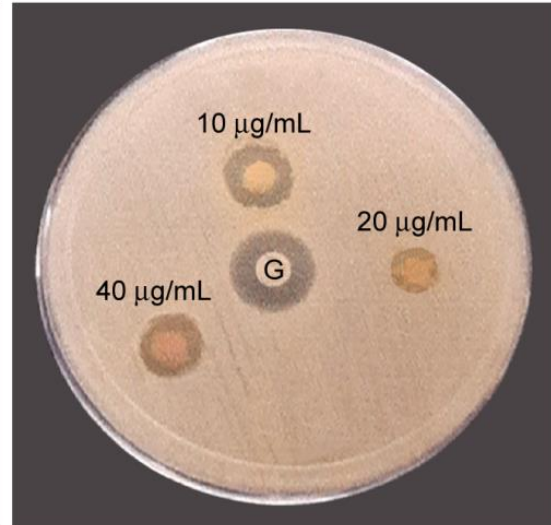

Staphylococcus aureus

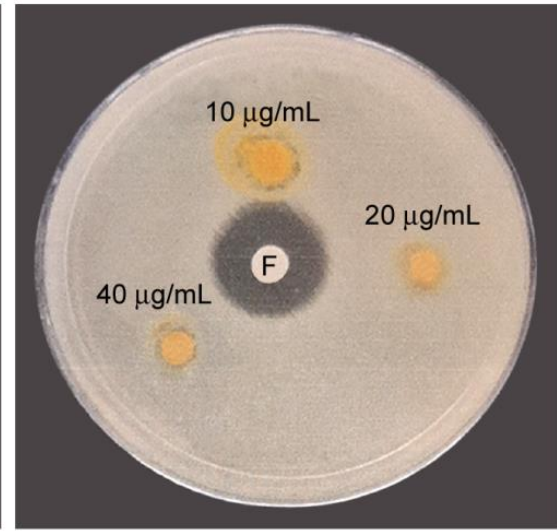

Aspergillus flavus

Figure 11. Antibacterial and antifungal activities of 2 .

The remaining substituted chalcones were satisfactory activity for the strain. Antifungal activity data (Table 8) if could be observed that all the chalcones 1-7 are a good activity for strain Candida albicans and moderate activity for Aspergillus flavus strains.

\section{Conclusions}

The known substituted chalcones 1-7 were characterized by using experimental and computational methods. The molecular properties such as bond length and bond angles were theoretically investigated by the optimized structure, and favored conformation of chalcone 3 was investigated. The nature and origin of the intramolecular electrons transition of HOMOLUMO optimized structure were derived from all the synthesized compounds. The electrophilic and nucleophilic reactive sites probable binding sites of the compounds were obtained by using MEP surface analysis. The polarizability and hyperpolarizability values were calculated, and NLO activity was determined by the substituted chalcones $1-7$. The Mulliken charges, NBO, natural charges, ${ }^{1} \mathrm{H}$, and ${ }^{13} \mathrm{C}$ NMR values were also compared experimentally and theoretically. The antibacterial and antifungal activity was derived by the disc diffusion method. All the substituted chalcones were good active against corresponding strains.

\section{Funding}

This research received no external funding.

\section{Acknowledgments}

The authors thank the NMR Research Centre, SAIF, Cochin, for recording the NMR spectra. 


\section{Conflicts of Interest}

The authors declare no conflict of interest.

\section{References}

1. Avila, H.P; Smania, E.F.A.; Monache, F.D.; Smania, J.A. Structure-activity relationship of antibacterial chalcones. Bioorganic and medicinal chemistry 2008, $16 \quad 9790-9794$, https://doi.org/10.1016/j.bmc.2008.09.064.

2. Cabrera M. Simoens M, Falchi G, Lavaggi M.L, Piro, O.E.; Castellano, E.E.; Vidal, A.; Azqueta, A.; Monge, A.; Cerain, A.Ld.; Sagrera, G.; Seoane, G.; Cerecetto, H.; Gonzalez, M. Synthetic chalcones, flavanones, and flavones as antitumoral agents: biological evaluation and structure-activity relationships. Bioorganic and medicinal chemistry 2007, 15, 3356-3367, https://doi.org/10.1016/j.bmc.2007.03.031.

3. Chiaradia, L.D.; Santos R dos; Victor, C.E.; Vieira, A.A.; Leal, P.C.; Nunes, R.J.; Calixto, J.B.; Yunes, R.A. Synthesis and pharmacological activity of chalcones derived from 2,4,6-trimethoxyacetophenone in RAW 264.7 cells stimulated by LPS: Quantitative structure-activity relationships. Bioorganic and medicinal chemistry 2008, 16, 658-667, https://doi.org/10.1016/j.bmc.2007.10.039.

4. $\quad$ 4. Teixeira, A.M.R.; Santos, H.S.; Bandeira, P.N.; Julião, M.S.S.; Freire, P.T.C.; Lima, V.N.; Cruz, B.G.; da Silva, P.T.; Coutinho, H.D.M.; Sena, D.M. Structural, spectroscopic and microbiological characterization of the chalcone 2E-1-(2'-hydroxy-3',4',6'trimethoxyphenyl)-3-(phenyl)-prop-2-en-1-one derived from the natural product 2-hydroxy-3,4,6-trimethoxyacetophenone. Journal of molecular structure 2019, 1179, 739748, https://doi.org/10.1016/j.molstruc.2018.11.075.

5. Freitas, T.S.D.; Xavier, J.D.C.; Pereira, R.L.S.; Rocha, J.E.; Muniz, D.F.; da Silva, P.T.; da Hora, J.P.; dos Santos, H.S.; Bandeira, P.N.; Nogueira, C.E.S.; Teixeira, A.M.R.; Coutinho, H.D.M. Direct antibacterial and antibiotic resistance modulatory activity of chalcones synthesized from the natural product 2-hydroxy-3,4,6trimethoxyacetophenone. FEMS microbiology letters 2020, 367, http://dx.doi.org/10.1093/femsle/fnaa124.

6. Silva, P.T.; Freitas, T.S.; Sena Jr., D.M.; Bandeira, P.N.; Julião, M.S.S.; Marinho, E.S.; Alcanfor, A.A.C.; Marinho, E.M.; Lima-Neto, P.; Nogueira, C.E.S.; Coutinho, H.D.M.; Leal, A.L.A.B.; Barreto, H.M.; Martins, N.; Rodrigues Teixeira, A.M.; Santos, H.S. Structural, vibrational and electrochemical analysis and antibacterial potential of isomeric chalcones derived from natural acetophenone. Applied Sciences 2020, 10, 4713, http://dx.doi.org/10.3390/app10144713.

7. Vagdevi, H.M., Latha, K.P., Vaidya, V.P., Kumar, M.L.V., Pai, K.S.R. Synthesis and pharmacological screening of some novel naphtho [2,1-b] furo-pyrazolines, isoxazoles and isoxazolines. Indian journal of pharmaceutical sciences 2001, 63, 286-291.

8. Pandya, M.; Kapadiya, K.; Pandit, C.; Purohit, D. Synthesis of halogenated chalcones pyrazolines and microbial evaluation of derived scaffolds. Journal of scientific and industrial research 2017, 76, 173-178.

9. Ashraf, S.; Hameed, S.; Tahir, M.N.; Naseer, M.M. Synthesis and crystal structure of bis-chalcone-derived fused-ring pyrazoline having an unusual substitution pattern, Monatsh chem 2017, 148, 1871-1875, https://doi.org/10.1007/s00706-017-1995-8.

10. Ahmed, M.; Hameed, S.; Ihsan, A.; Naseer, M.M. Fluorescent thiazol-substituted pyrazoline nanoparticles for sensitive and highly selective sensing of explosive 2,4,6-trinitrophenol in aqueous medium. Sens actuators B 2017, 248, 57-62, https://doi.org/10.1016/j.snb.2017.03.125.

11. Abbas, A.; Holguin, N.F.; Naseer, M.M. Structure-fluorescence relationship: interplay of non-covalent interactions in homologous 1,3,5-triaryl-2-pyrazolines. New journal of chemistry 2015, 39, 4359-4367. https://doi.org/10.1039/C5NJ00179J.

12. Anum, F.; Abbas, A.; Lo, K.M.; Rehman, Z.U.; Hameed, S.; Naseer, M.M. Homologous 1,3,5triarylpyrazolines: synthesis, $\mathrm{CH} \cdots \pi$ interactions guided self-assembly and effect of alkyloxy chain length on DNA binding properties. New journal of chemistry 2014, 38, 5617-5625, https://doi.org/10.1039/C4NJ00938J.

13. Abbas, A.; Naseer, M.M. Synthesis and anti-inflammatory activity of new N-Acyl-2-pyrazolines bearing homologous alkyloxy side chains. Acta chimica slovenica 2014, 61, 792-802.

14. Samshuddin, S.; Narayana, B.; Sarojini, B.K.; Shetty, D.N.; Suchetha Kumari, N. Synthesis, characterization, and biological evaluation of some new functionalized terphenyl derivatives. International journal of medicinal chemistry 2012, 2012, 1-13, https://doi.org/10.1155/2012/530392.

15. Bhat, B.A.; Dhar, K.L.; Puri, S.C.; Saxena, A.K.; Shanmugavel, M.; Qazi, G.N. Synthesis and biological evaluation of chalcones and their derived pyrazoles as potential cytotoxic agents. Bioorganic \& medicinal chemistry letters 2005, 15, 3177-3180, https://doi.org/10.1016/j.bmcl.2005.03.121.

16. Monga, V.; Goyal, K.; Steindel, M.; Malhotra, M.; Rajani, D.P.; Rajani, S.D. Synthesis and evaluation of new chalcones, derived pyrazoline and cyclohexenone derivatives as potential antimicrobial, antitubercular and antileishmanial agents. Medicinal chemistry research 2014, 23, 2019-2032, https://doi.org/10.1007/s00044-013-0803-1. 
17. Arora, P.K.; Mittal, A.; Kaur, G.; Chauhan, A. Synthesis of some novel oxadiazole based chalcone derivatives as antibacterial agents. International journal of pharmaceutical sciences and research 2013, 4, 419-424, http://dx.doi.org/10.13040/IJPSR.0975-8232.

18. Demet Coskun; Bayram Gunduz; Mehmet Fatih Coskun. Synthesis, characterization and significant optoelectronic parameters of 1-(7-methoxy-1-benzofuran-2-yl) substituted chalcone derivatives Journal of molecular structure 2019, 1178, 261-267, https://doi.org/10.1016/j.molstruc.2018.10.043.

19. Hegde, H; Sinha, R. K; Kulkarni, S. D; Nitinkumar, S; Shetty, N.S. Synthesis, photophysical and DFT studies of naphthyl chalcone and nicotinonitrile derivatives. Journal of Photochemistry and Photobiology A: Chemistry 2020, 389, https://doi.org/10.1016/j.jphotochem.2019.112222.

20. Aboelnaga,A.; Eman Mansour, E.; Hoda, A.; Ahmed, H.M. Synthesis of Asymmetric Pyrazoline Derivatives from Phenylthiophenechalcones; DFT Mechanistic Study. Journal of the Korean Chemical Society 2021, 65, https://doi.org/10.5012/jkcs.2021.65.2.113.

21. Uchida, T.; Kozawa, K.; Sakai, T.; Aoki, M.; Yoguchi, H.; Abdureyim, A.; Watanabe, Y. Novel Organic SHG Materials. Molecular Crystals and Liquid Crystals Science and Technology. Section A. Molecular Crystals and Liquid Crystals 1998, 315, 135-140, https://doi.org/10.1080/10587259808044322.

22. Indira, J.; Karat, P.P.; Sarojini, B.K. Growth, characterization and non-linear optical property of chalcone derivative. Journal of Crystal Growth 2002, 242, 209-214, https://doi.org/10.1016/S0022-0248(02)01306-4.

23. Tien, C.N.; Quoc, T.V.; Dang, N.D.; Duc, G.L.; Meervelt, L.V Synthesis and structure of (E)-N-(4methoxyphenyl)-2-[4-(3-oxo-3-phenylprop-1-en-1-yl)phenoxy]-acetamide. Acta crystallographica 2021, 77, 184-189, https://doi.org/10.1107/S2056989021000864.

24. Khairul, W.M.; Daud, A.I.; Augustine, E.; Arshad, S.; Razak, I.A. FT-IR, NMR and X-ray crystallography dataset for newly synthesized alkoxy-chalcone featuring (E)-1-(4-ethylphenyl)-3-(4-(heptyloxy) phenyl)prop-2-en-1-one. Chemical Data Collections 2020, 28, https://doi.org/10.1016/j.cdc.2020.100473.

25. Custodio, J.M.F.; D’Oliveira, G.D.C.; Gotardo, F.; Cocca, L.H.Z.; Boni, L. de.; Perez, C.N.; Napolitano, H.B.; Osorio F.A.P.; Valverde. C. Second-order non-linear optical properties of two chalcone derivatives: insights from sum-over-states. Physical chemistry chemical physics 2021, 23, 6128-6140, https://doi.org/10.1039/D0CP06469F.

26. Shruthi, C.; Ravindrachary, V.; Guruswamy, B.; Prasad, D.J.; Goveas, J.; Kumara, K.; Lokanath, N.K. Molecular structure, Hirshfeld surface and density functional theoretical analysis of a NLO active chalcone derivative single crystal-A quantum chemical approach. Journal of Molecular Structure 2021, 1228, https://doi.org/10.1016/j.molstruc.2020.129739.

27. Madan, N.; Sunil, K; Yamuna, I.K.; Chethan Prathap, K.N.; Lokanath, N.K. Structural investigation, hirshfeld surface analysis and dft calculations of (e)-3-(2-chlorophenyl)-1-(2-fluoro-4- methoxyphenyl) prop-2-en-1one. Journal of critical reviews 2020, 7, 3754-3763.

28. Day, P.N.; Pachter, R.; Nguyen, K.A. Analysis of non-linear optical properties in donor-acceptor materials. Journal of chemical physics 2014, 140, https://doi.org/10.1063/1.4874267.

29. Abegão, L.M.G.; Fonseca, R.D.; Ramos, T.N.; Mahuteau-Betzer, F.; Piguel, S.; Joatan R, J.; Mendonça, C.R.; Canuto, S.; Silva, D.L.; De Boni, L. Oxazole Dyes with Potential for Photoluminescence Bioprobes: A TwoPhoton Absorption Study. The Journal of Physical Chemistry C 2018, 122, 10526-10534, https://doi.org/10.1021/acs.jpcc.8b01904.

30. Santos, F.A.; Abegão, L.M.G.; Fonseca, R.D.; Alcântara, A.M.; Mendonça, C.R.; Valle, M.S.; Alencar, M.A.R.C.; Kamada, K.; De Boni, L.; Rodrigues, J.J. Bromo-and chloro-derivatives of dibenzylideneacetone: Experimental and theoretical study of the first molecular hyperpolarizability and two-photon absorption. Journal of Photochemistry and Photobiology A: Chemistry 2019, 369, 70-76, https://doi.org/10.1016/j.jphotochem.2018.10.012.

31. Anam, F.; Abbas, A.; Lo, K.M.; Zia ur, R.; Hameed, S.; Naseer, M.M. Homologous 1,3,5-triarylpyrazolines: synthesis, $\mathrm{CH} \cdots \pi$ interactions guided self-assembly and effect of alkyloxy chain length on DNA binding properties. New Journal of Chemistry 2014, 38, 5617-5625, https://doi.org/10.1039/C4NJ00938J.

32. Balachander, R.; Manimekalai, A. Synthesis, conformational and theoretical studies of 1,n-di(2-formyl-4phenylazophenoxy)alkanes. Journal of Molecular Structure 2016, 1104, 70-78, https://doi.org/10.1016/j.molstruc.2015.10.006.

33. Renuka, V.; Revathi, B.K.; Jonathan, D.R.; Priya, M.K.; Asirvatham, P.S. Synthesis, growth and characterization of a new NLO active chalcone derivative - 4-chloro-N-\{3-[(2E)-3-(methoxyphenyl)prop-2Enoyl]phenyl\}benzamide monohydrate. Journal of Molecular Structure 2019, 1176, 838-846, https://doi.org/10.1016/j.molstruc.2018.09.021.

34. Patil, P.S.; Maidur, S.R.; Shkir, M.; AlFaify, S.; Ganesh, V.; Krishnakanth, K.N.; Rao, S.V. Crystal growth and characterization of second- and third-order non-linear optical chalcone derivative: (2E)-3-(5-bromo-2thienyl)-1-(4-nitrophenyl)prop-2-en-1-one. Jouranl of appllied crystallography 2018, 51, 1035-1042, https://doi.org/10.1107/S1600576718006386.

35. Muhammad, S.; Al-Sehemi, A.G.; Irfan, A.; Algarni, H.; Qiu, Y.; Xu, H.; Su, Z.; Iqbal, J. The substitution effect of heterocyclic rings to tune the optical and non-linear optical properties of hybrid chalcones: A comparative study. Journal of Molecular Graphics and Modelling 2018, 81, 25-31, https://doi.org/10.1016/j.jmgm.2018.02.005. 
36. Borges, I.D.; Danielli, J.A.V.; Silva, V.E.G.; Sallum, L.O.; Queiroz, J.E.; Dias, L.D.; Iermak, I.; Aquino, G.L.B.; Camargo, A.J.; Valverde, C.; Osório, F.A.P.; Baseia, B.; Napolitano, H.B. Synthesis and structural studies on (E)-3-(2,6-difluorophenyl)-1-(4-fluorophenyl)prop-2-en-1-one: a promising non-linear optical material. RSC Advances 2020, 10, 22542-22555, https://doi.org/10.1039/d0ra03634j.

37. Wijayanti, L.W.; Swasono, R.T.; Lee, W.; Jumina, J. Synthesis and Evaluation of Chalcone Derivatives as Novel Sunscreen Agent. Molecules 2021, 26, https://doi.org/10.3390/molecules26092698.

38. Twilley, D.; Moodley, D.; Rolfes, H.; Moodley, I.; McGaw, L.J.; Madikizela, B.; Summers, B.; Raaff, L.-a.; Lategan, M.; Kgatuke, L.; Mabena, E.C.; Lall, N. Ethanolic extracts of South African plants, Buddleja saligna Willd. and Helichrysum odoratissimum (L.) Sweet, as multifunctional ingredients in sunscreen formulations. South African Journal of Botany 2021, 137, 171-182, https://doi.org/10.1016/j.sajb.2020.10.010.

39. Bhattacharya, S.; Sherje, A.P. Development of resveratrol and green tea sunscreen formulation for combined photoprotective and antioxidant properties. Journal of Drug Delivery Science and Technology 2020, 60, 16. https://doi.org/10.1016/j.jddst.2020.102000.

40. Amador-Castro, F.; Rodriguez-Martinez, V.; Carrillo-Nieves, D. Robust natural ultraviolet filters from marine ecosystems for the formulation of environmental friendlier bio-sunscreens. Science of The Total Environment 2020, 749, https://doi.org/10.1016/j.scitotenv.2020.141576.

41. Wang, G.; Liu, W.; Gong, Z.; Huang, Y.; Li, Y.; Peng, Z. Design, synthesis, biological evaluation and molecular docking studies of new chalcone derivatives containing diaryl ether moiety as potential anticancer agents and tubulin polymerization inhibitors. Bioorganic chemistry 2020, 95.

42. Qin, H.-L.; Zhang, Z.-W.; Lekkala, R.; Alsulami, H.; Rakesh, K.P. Chalcone hybrids as privileged scaffolds in antimalarial drug discovery: A key review. European Journal of Medicinal Chemistry 2020, 193, https://doi.org/10.1016/j.ejmech.2020.112215.

43. Janković, T.; Turković, N.; Kotur-Stevuljević, J.; Vujić, Z.; Ivković, B. Differences in antioxidant potential of chalcones in human serum: In vitro study. Chemico-biological interactions 2020, 324, https://doi.org/10.1016/j.cbi.2020.109084.

44. Shunmuga Vadivoo, V.; Mythili, C.V.; Vijaya, P. DFT computational survey of antimicrobial 1,3-bis(4methoxyphenyl)prop-2-en-1-one. ADALYA Journal 2020, 9, 295-305.

45. Frisch, M.J.; Trucks, G.W.; Schlegel, H.B.; Scuseria, G.E.; Robb, M.A.; Cheeseman, J.R.; Zakrzewski, V.G.; Montgomery Jr., J.A.; Stratmann, R.E.; Burant, J.C.; Dapprich, S.; Millam, J.M.; Daniels, A.D.; Kudin, K.N.; Strain, M.C.; Farkas, O.; Tomasa, J.; Barone, V.; Cossi, M.; Cammi, R.; Mennucci, B.; Pomeli, C.; Adamo, C.; Clifford, S.; Ochterski, J.; Peterson, G.A.; Ayala, P.Y.; Cui, Q.; Morokuma, K.; Salvador, P.; Dannenberg, J.J.; Malick, D.K.; Rabuck, A.D.; Raghavachari, K.; Poresman, J.B.; Cioslowski, J.; Ortiz, J.N.; Babboul, A.G.; Stefavov, B.B.; Liu, G.; Liashenko, A.; Piskorz, P.; Komaromi, I.; Gomperts, R.; Martin, R.L.; Fox, D.J.; Keeth, T.; Allaham, M.A.; Peng, C.Y.; Nanayakkara, A.; Wong, M.W.; Anders, J.L.; Gonzales, C.; Challacombe, M.; Gill, P.M.; Johnson, B.; Chen, W.; Head-Gordon, M.; Replogle, E.S.; Peple, J.A. Gaussian 98, Revision A.9, Pittsburg, Pa: Gaussian IC, 2001. Revision A.9, Pittsburg, Pa: Gaussian IC. Reference to a chapter in an edited book.

46. Crasta, V.; Ravindrachary, V.; Bhajantri, R.F.; Gonsalves, R. Growth and characterization of an organic NLO crystal: 1-(4-methylphenyl)-3-(4-methoxyphenyl)-2-propen-1-one. Journal of crystal growth 2004, 267, 129-133, https://doi.org/10.1016/j.jcrysgro.2004.03.037.

47. Öztürk, N.; Özdemir, T.; Alpaslan, Y.B.; Gökce, H.; Alpaslan, G. Experimental (FT-IR, Raman and NMR) and theoretical (B3LYP, B3PW91, M06-2X and CAM-B3LYP) analyses of p-tertbutylphenyl salicylate, Bilge. International journal of science and technology research 2018, 2, 56-73, https://doi.org/10.30516/bilgesci.354763.

48. Hugas, D.; Simon, S.; Duran, M. Electron density topological properties are useful to assess the diference between hydrogen and dihydrogen complexes. The journal of physical chemistry A 2007, 111, 4506-4512, https://doi.org/10.1021/jp070080u.

49. Balachander, R.; Manimekalai, A. Synthesis, spectral and computational analysis of 2-(N-bromoalkoxy)-5(phenylazo) benzaldehydes and their oximes. Journal of molecular Structure 2017, 1134, 552-562, https://doi.org/10.1016/j.molstruc.2017.01.018.

50. Bauer, A.W.; Kirby, W. M. ; Sherris, J.C.; Turck, M. Antibiotic susceptibility testing by a standardized single disk method. American journal of clinical pathology 1966, 45, 493-496.

51. Xu, M.; Wu, P.; Shen, F.; Ji, J.; Rakesh, K.P. Chalcone derivatives and their antibacterial activities: Current development. Bioorganic Chemistry 2019, 91, https://doi.org/10.1016/j.bioorg.2019.103133.

52. da Silvaa, P.T.; da Cunha Xavier, J.; Freitas, T.S.; Oliveira, M.M.; Coutinho, H.D.M.; Leal, A.L.A.B.; Barreto, H.M.; Bandeira, P.N.; Nogueiraa, C.E.S.; Sena Jr. D.M.; Almeida-Netoe, F.W.Q.; Marinhof, E.S.; Santos, H.S.; Teixeiraa, A.M.R. Synthesis, spectroscopic characterization and antibacterial evaluation by chalcones derived of acetophenone isolated from Croton anisodontus Müll.Arg. Journal of molecular structure 2021, 1226, https://doi.org/10.1016/j.molstruc.2020.129403. 